Article

\title{
New Uncharged 2-Thienostilbene Oximes as Reactivators of Organophosphate-Inhibited Cholinesterases
}

\author{
Milena Mlakić ${ }^{1,+}$, Tena Čadež ${ }^{2,+}$, Danijela Barić ${ }^{3} \mathbb{D}$, Ivana Puček ${ }^{1}$, Ana Ratković ${ }^{4}$, Željko Marinić ${ }^{5}$ (D), \\ Kornelija Lasić ${ }^{6}$ (D), Zrinka Kovarik ${ }^{2, *(D)}$ and Irena Škorić 1,*(D)
}

1 Department of Organic Chemistry, Faculty of Chemical Engineering and Technology, University of Zagreb, Marulićev trg 19, HR-10 000 Zagreb, Croatia; mdragojev@fkit.hr (M.M.); ivana.pucek812@gmail.com (I.P.)

2 Institute for Medical Research and Occupational Health, Ksaverska cesta 2, HR-10 000 Zagreb, Croatia; tcadez@imi.hr

3 Group for Computational Life Sciences, Division of Physical Chemistry, Ruđer Bošković Institute, Bijenička cesta 54, HR-10 000 Zagreb, Croatia; dbaric@irb.hr

4 Fidelta Ltd., Prilaz Baruna Filipovića 29, HR-10 000 Zagreb, Croatia; ana.ratkovic30@gmail.com

5 NMR Center, Ruđer Bošković Institute, Bijenička cesta 54, HR-10 000 Zagreb, Croatia; zmarinic@irb.hr

6 Pliva Tapi R\&D, TEVA, Prilaz baruna Filipovića 25, HR-10 000 Zagreb, Croatia; Kornelija.Lasic@pliva.com

* Correspondence: zkovarik@imi.hr (Z.K.); iskoric@fkit.hr (I.Š.)

+ These authors contributed equally.

Citation: Mlakić, M.; Čadež, T.;

Barić, D.; Puček, I.; Ratković, A.;

Marinić, Ž.; Lasić, K.; Kovarik, Z.;

Škorić, I. New Uncharged

2-Thienostilbene Oximes as

Reactivators of Organophosphate-

Inhibited Cholinesterases.

Pharmaceuticals 2021, 14, 1147.

https://doi.org/10.3390/

ph14111147

Academic Editors: Urszula

K. Komarnicka, Monika Lesiów and Sabina Jaros

Received: 25 October 2021

Accepted: 8 November 2021

Published: 11 November 2021

Publisher's Note: MDPI stays neutral with regard to jurisdictional claims in published maps and institutional affiliations.

Copyright: (c) 2021 by the authors. Licensee MDPI, Basel, Switzerland. This article is an open access article distributed under the terms and conditions of the Creative Commons Attribution (CC BY) license (https:/ / creativecommons.org/licenses/by/ $4.0 /)$.

\begin{abstract}
The inhibition of acetylcholinesterase (AChE) and butyrylcholinesterase (BChE) by organophosphates (OPs) as nerve agents and pesticides compromises normal cholinergic nerve signal transduction in the peripheral and central nervous systems (CNS) leading to cholinergic crisis. The treatment comprises an antimuscarinic drug and an oxime reactivator of the inhibited enzyme. Oximes in use have quaternary nitrogens, and therefore poorly cross the brain-blood barrier. In this work, we synthesized novel uncharged thienostilbene oximes by the Wittig reaction, converted to aldehydes by Vilsmeier formylation, and transformed to the corresponding uncharged oximes in very high yields. Eight trans,anti- and trans,syn-isomers of oximes were tested as reactivators of nerve-agent-inhibited AChE and BChE. Four derivatives reactivated cyclosarin-inhibited $\mathrm{BChE}$ up to $70 \%$ in two hours of reactivation, and docking studies confirmed their productive interactions with the active site of cyclosarin-inhibited BChE. Based on the moderate binding affinity of both $\mathrm{AChE}$ and $\mathrm{BChE}$ for all selected oximes, and in silico evaluated ADME properties regarding lipophilicity and CNS activity, these compounds present a new class of oximes with the potential for further development of CNS-active therapeutics in OP poisoning.
\end{abstract}

Keywords: AChE; BChE; reactivation; heterostilbenes; spectroscopy; docking

\section{Introduction}

Oximes, unlike the symptomatic drugs currently used as inhibitors of acetylcholinesterase (AChE) in the treatment of neurological disorders such as Alzheimer's and Parkinson's disease, have an important role for the regeneration of the cholinergic system in cases of poisoning with organophosphate compounds (OPs). OPs present a large group of chemicals used as pesticides, flame retardants, etc., and misused as nerve agents. Their non-selective species toxicity can cause severe biological effects to humans [1,2]. The related enzyme butyrylcholinesterase $(\mathrm{BChE})$ is also involved in the regulation of the cholinergic system as a backup to AChE in hydrolyzing the neurotransmitter acetylcholine (ACh) in neurological disorders [3]. BChE's role also seems to be the protection of synaptic AChE because it reacts with a variety of drugs, xenobiotics as well as with OPs, but its inhibition is safe for human health [4,5]. Emergency treatment for AChE inhibited with OPs is based on oxime reactivators that regenerate the enzyme through the nucleophilic displacement of the OP moiety conjugated at the catalytic center serine. Oxime therapy comprises anticonvulsants and antimuscarinics to manage symptoms prompted by poisoning manifested as salivation, tremors, respiratory paralysis, and in severe exposures, death [6,7]. Unfortunately, the current standard therapy in OP 
poisoning is still based on a small number of quaternary pyridinium compounds, 2-PAM, obidoxime, or HI-6, which are not universal reactivators due to the structural variability of OPs and their structural impotence in passing the brain-blood barrier (BBB) [7-10].

To improve the central nervous system (CNS) activity of oximes, our recent research was directed towards oximes with increased lipophilicity, uncharged oximes, and zwitterionic oximes with a tertiary nitrogen atom $[9,10]$. Even though uncharged oximes were usually proven to be much better in BBB penetration due to increased lipophilicity [11,12], their downfall is the low affinity as a consequence of the absence of a positive charge for productive interactions with the OP-inhibited enzyme $[13,14]$. This is where the concept of a peripheral site ligand (PSL) was introduced into the oxime structure as compensation for the positively charged nitrogen in order to preserve, if not increase, the affinity for the enzyme $[9,10,15,16]$. The introduction of the PSL strategy, which involves the coupling of uncharged pyridine oximes with different PSLs via alkyl chains, has opened up many options for new oxime structures.

In this paper, we describe the synthesis of a new uncharged thienostilbene oxime whose design was based on our long-term experience in the preparation of heterostilbenes with thiophene and furan nuclei [17]. Moreover, a study on (thiophen-2-yl)-aldoximes reported that the sulfur atom, due to its potential for polarization, helps to stabilize the negative charge of its anionic form, similarly to the pyridinium ring of the standard oxime 2-PAM [18]. Therefore, we present here the preparation of a five-membered heterocyclic thiophene nucleus connected by a double bond to the benzene ring with para-position substitution that could be accommodated at the peripheral site and improve binding affinity. It is worth mentioning that the double-bond introduction between two (hetero)aryl groups eliminates the possibility of a pronounced conformational change. Nevertheless, a new oxime group was obtained by an efficient and cost-effective reaction pathway involving the Wittig reaction, Vilsmeier formylation, and the formation of two configurationally stable oxime isomers. We evaluated the kinetic interaction of each isomer with both $\mathrm{AChE}$ and $\mathrm{BChE}$, together with their reactivation potential in sarin, cyclosarin, tabun, and VX poisoning. An in silico analysis of basic physicochemical characteristics was performed to estimate the ability of novel oximes in BBB crossing, as well as to assess their potential for further biological testing.

\section{Results and Discussion}

\subsection{Synthesis and Spectroscopic Characterization of New Thienostilbene Oximes 13-18}

The preparation of the desired thienostilbene oximes 13-18 was achieved according to the three-step reaction path shown in Scheme 1. It began by forming a double bond using the Wittig reaction to yield the starting compounds $\mathbf{1 - 6}$ as mixtures of cis- and trans-isomers (48-61\%). In the second step, Vilsmeier formylation provided the corresponding mixtures of aldehydes 7-12 (24-47\%) and in the third step, formyl derivatives were transformed to oximes 13-18 (57-88\%) as the targeted structures. Mixtures of the geometrical isomers of oximes 13-18 were separated to obtain pure compounds by repeated-column and thin-layer chromatography.

The Wittig reaction was performed using commercially available thiophene-2carbaldehyde, and selected phosphonium salts prepared in our laboratory. The obtained thienostilbenes 1-6 represent the basic building blocks subjected to the Vilsmeier formylation reaction. In this reaction, $\mathrm{POCl}_{3}$ and DMF initially formed a chloriminium ion, a Vilsmeier reagent, which then reacted with the heterostilbene molecule, and an iminium ion was formed, which hydrolyzed to the final aldehydes 7-12. We noticed that the trans-isomers of 1-6 reacted more successfully in the formylation carried out for the partially unreacted cis-isomers, and thus the proportion of trans-isomers of aldehydes 7-12 was significantly higher (18-30\%) in comparison to those for the cis-isomers (1-19\%). The reaction of converting the synthesized aldehydes 7-12 into the corresponding oximes 13-18 involved the use of $\mathrm{NH}_{2} \mathrm{OH} \times \mathrm{HCl}$ and a mixture of ethanol and water as solvents and was based on the reaction described in the literature on simpler heteroaromatic systems [18]. According to ${ }^{1} \mathrm{H}$ NMR spectroscopic analyses, 
trans-isomers are more present in the reaction mixtures of oxime derivatives 13-18 (Figure 1). In the case of oximes 13-15 and 18 possessing $\mathrm{CH}_{3}, \mathrm{OCH}_{3}$, and $\mathrm{Cl}$ groups as substituents, respectively, trans, anti-isomers dominate. Oximes 16 and $\mathbf{1 7}$ with electron-withdrawing substituents $\mathrm{CN}$ and $\mathrm{NO}_{2}$, respectively, have the most trans,syn-isomers in the reaction mixture, while in the case of the $\mathrm{N}\left(\mathrm{CH}_{3}\right)_{2}$ substituent, the ratio of $s y n$ - and anti-isomers was 2:1, with the presence of cis,syn-18 at the same abundance as that of trans,syn-18 (See Experimental Section) [19].

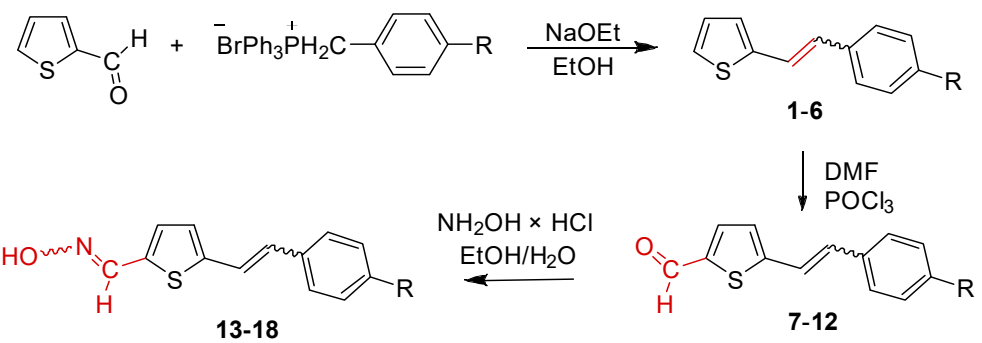

13: $\mathrm{R}=\mathrm{CH}_{3}(87 \%) ; 14: \mathrm{R}=\mathrm{OCH}_{3}(83 \%)$

15: $\mathrm{R}=\mathrm{Cl}(77 \%) ; 16: \mathrm{R}=\mathrm{CN}(73 \%)$
17: $\mathrm{R}=\mathrm{NO}_{2}(57 \%) ; \mathbf{1 8}: \mathrm{R}=\mathrm{N}\left(\mathrm{CH}_{3}\right)_{2}(88 \%)$

Scheme 1. The three-step reaction pathway for obtaining heterostilbene oxime derivatives 13-18. The yields are expressed for the third reaction step.

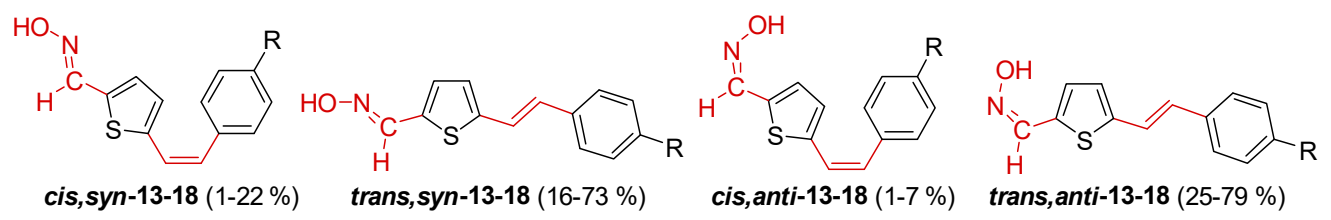

Figure 1. Ratio of geometrical isomers of the synthesized thienostilbene oximes 13-18.

After successive column and thin-layer chromatography, the trans,anti- and trans, syn-isomers of the individual oxime derivatives 13-18 were isolated at a sufficient quantity to test them as potential cholinesterase reactivators (see Section 2.3). During the experiment, it was found that the anti-isomers of oximes are more polar and less soluble than those of syn-isomers, and due to the difference in their $R_{f}$ values, they are relatively easily isolated. The similarity of the $\mathrm{R}_{f}$ values of the cis- and trans-isomers of the syn-oximes influenced the lower success of their separation. All of the isolated isomers of oxime 13-18 were fully spectroscopically characterized (See Figures 2 and 3, Experimental Section and Supplementary Materials).

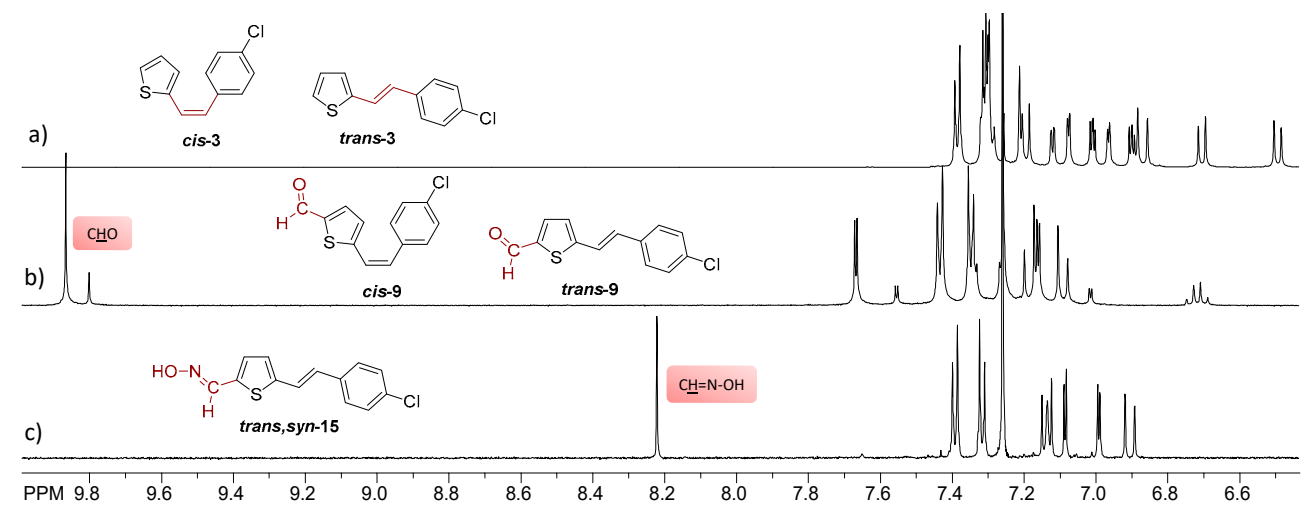

Figure 2. Downfield region of the ${ }^{1} \mathrm{H}$ NMR spectra $\left(\mathrm{CDCl}_{3}\right)$ of the mixture of cis-3 and trans-3 (a), mixture of cis-9 and trans-9 (b), and oxime trans,syn-15 (c). 


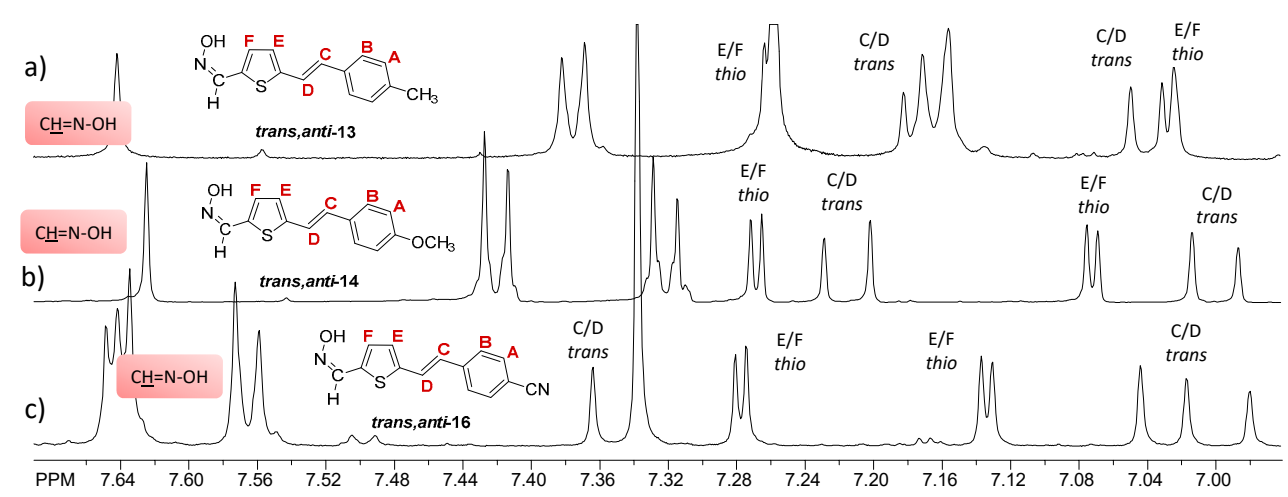

Figure 3. Downfield region of the ${ }^{1} \mathrm{H} N M R$ spectra $\left(\mathrm{CDCl}_{3}+\mathrm{CD}_{3} \mathrm{OD}\right)$ of the oximes trans, anti-13 (a), trans, anti-14 (b), and trans, anti-16 (c).

Additionally, from the reaction mixtures after the Wittig and Vilsmeier reaction, cisand trans-isomers of thienostilbene 1-6 as well as the cis- and trans-isomers of the formylation products 7-12 were spectroscopically characterized and given for analysis by HRMS (See Experimental Section and Supplementary Materials).

\subsection{In Silico Prediction of ADME Properties of Thienostilbene Oximes 13-18}

The structure-property relationships [20,21] with predictive models for calculated compounds 13-18 were also analyzed by ACD/Percepta (ver. 14.2.0; Build 2977; ACD/Laboratories, Toronto, ON, Canada), which does not consider the geometrical isomerization. Physicochemical properties were calculated, specifically the $\log \mathrm{P}$ values, solubility, and permeability across biological membranes (Table 1). From a medicinal chemistry perspective, the design of drugs capable of penetrating the blood-brain barrier (BBB) and effecting the desired biological response is a formidable challenge. Moderately lipophilic drugs cross the BBB by passive diffusion, and the hydrogen bonding properties of drugs can significantly influence their CNS profiles. Polar molecules are generally poor CNS agents unless they undergo active transport across the CNS. Size, ionization properties, and molecular flexibility are other factors observed to influence the transport of an organic compound [22]. Lipophilicity is one of the most important physical properties that can affect the potency, distribution, and elimination of a drug in the body [23].

Table 1. ADME properties of the oxime derivatives 13-18.

\begin{tabular}{|c|c|c|c|c|c|}
\hline Compound & $\log P^{a}$ & $\begin{array}{l}\text { Solubility }^{\mathbf{b}} \\
{[\mathrm{mg} / \mathrm{mL}]}\end{array}$ & $\begin{array}{c}\text { Permeability }^{\mathrm{c}} \\
{\left[10^{-6} \mathrm{~cm} / \mathrm{s}\right]}\end{array}$ & PPB $^{d}$ & $\mathrm{CNS}^{\mathrm{e}}$ \\
\hline 13 & 3.95 & 0.004 & 23.6 & $83 \%$ & -1.86 \\
\hline 14 & 3.70 & 0.009 & 23.7 & $77 \%$ & -1.77 \\
\hline 15 & 4.27 & 0.002 & 23.3 & $90 \%$ & -2.05 \\
\hline 16 & 3.32 & 0.009 & 23.4 & $77 \%$ & -1.82 \\
\hline 17 & 3.58 & 0.01 & 23.6 & $81 \%$ & -1.92 \\
\hline 18 & 3.94 & 0.01 & 23.6 & $83 \%$ & -1.86 \\
\hline
\end{tabular}

a lipophilicity $\log \mathrm{P}(<-2.0$ very hydrophilic, $-2.0-(-1.0)$ hydrophilic, $-1.0-4.2$ optimal, $4.2-5.0$ lipophilic); ${ }^{b}$ solubility $(<0.01$ highly insoluble, $0.01-0.10$ insoluble, $>0.10$ soluble $) ;{ }^{c}$ Caco permeability $\left(<1.0 \times 10^{-6}\right.$ poorly permeable, $1.0-7.0 \times 10^{-6}$ moderately permeable, $>7.0 \times 10^{-6}$ highly permeable); ${ }^{\mathrm{d}} \mathrm{PPB}-$ plasma-protein binding (10-40\% weakly bound, $40-80 \%$ moderately, $80-90 \%$ strongly bound, $>90 \%$ extensively bound); ${ }^{\mathrm{e}} \mathrm{CNS}$ $<-3.5$ non-penetrant, $-3.50-(-3.0)$ weak penetrant, $>-3.0$ penetrant); (ACD/Percepta (ver. 14.2.0; Build 2977; ACD/Laboratories, Toronto, ON, Canada).

In general, the solubility of selected oximes 13-18 was low. Nitro derivative $\mathbf{1 7}$ and dimethylamino derivative 18 showed the highest solubility $(0.01 \mathrm{mg} / \mathrm{mL})$. All of the compounds showed an optimal lipophilicity value, meaning they should readily penetrate the $\mathrm{BBB}$, since the majority of CNS drugs have $\log \mathrm{P}$ within the range of 3.5 to 4.5 (ACD/Percepta (ver. 14.2.0; Build 2977; ACD/Laboratories, Toronto, ON, Canada). Moreover, estimations of the CNS factor displayed their high penetrant ability. Plasma 
protein binding (PPB) results showed high bioavailability and a long biological half-life because the bound portion may act as a reservoir from which the drug is slowly released in the unbound form to maintain equilibrium. Nevertheless, based on these in silico ADME analyses, chloro derivative 15 seems to be the one with the least-desired physicochemical properties for a CNS active drug, yet the solubility of all thienostilbenes require improvement in the future.

\subsection{In Vitro Evaluation of Thienostilbene Oximes 13-18 in Reactions with Cholinesterases}

Eight thienostilbene oxime derivatives 13-18 were tested as reversible inhibitors of $\mathrm{AChE}$ and $\mathrm{BChE}$, and dissociation constants are given in Table 2. Generally, all of the oximes were moderate reversible inhibitors of both enzymes with $K_{i}$ constants in the micromolar range. All of the oximes, except trans, anti-16, were more potent inhibitors of AChE than BChE. Out of all of the tested oximes, BChE also exhibited higher potency for cis,syn-18, which has nitrogen at the substituent, as well as for trans,anti-16. In the case of $\mathrm{AChE}$, it seems that the CN substituent affected the binding affinity, as $K_{\mathrm{i}}$ for trans,anti-16 is up to 10-fold higher than for the most potent inhibitor of AChE trans,syn-15. In addition, inhibition of AChE was uncompetitive for all compounds, except for trans,syn-15, while all of the compounds exhibited competitive binding for BChE.

Table 2. Dissociation constants, $K_{\mathrm{i}}(\mu \mathrm{M}) \pm \mathrm{SE}$, for reversible inhibition of human acetylcholinesterase $(\mathrm{AChE})$ and butyrylcholinesterase $(\mathrm{BChE})$ with new oximes at $25^{\circ} \mathrm{C}$.

\begin{tabular}{ccc}
\hline Thienostilbene Oximes & AChE & BChE \\
\hline trans,syn-13 & $46 \pm 7$ & $355 \pm 26$ \\
trans,anti-13 & $41 \pm 4$ & $322 \pm 38$ \\
trans,syn-14 & $67 \pm 12$ & $271 \pm 24$ \\
trans,anti-14 & $54 \pm 7$ & $172 \pm 14$ \\
trans,syn-15 & $52 \pm 12$ & $573 \pm 35$ \\
trans,anti-15 & $76 \pm 21$ & $482 \pm 45$ \\
trans,anti-16 & $158 \pm 40$ & $44 \pm 9$ \\
cis,syn-18 & $39 \pm 11$ & $89 \pm 16$ \\
\hline
\end{tabular}

The reactivation of human $\mathrm{AChE}$ and $\mathrm{BChE}$ inhibited with nerve agents (sarin, cyclosarin, VX, and tabun) was screened with one concentration of thienostilbene oxime derivatives $(0.1 \mathrm{mM})$. In the case of AChE inhibited with any of the four nerve agents, no reactivation higher than $20 \%$ was observed within $5 \mathrm{~h}$, which does not present an improvement over TMB-4, 2-PAM, HI-6, and obidoxime in the case of AChE inhibited with tabun, VX, sarin, and cyclosarin, respectively. Unfortunately, reactivation could not be tested with a higher concentration due to poor solubility and solvent DMSO, which affects AChE activity. In the case of BChE, only the cyclosarin-inhibited BChE was prone to reactivation, and Figure 4 shows the percentage of maximal reactivation and the observed reactivation rate with $0.1 \mathrm{mM}$ oximes. The fastest reactivation was observed with trans, anti$\mathbf{1 5}$ and trans,anti-14, while with their trans,syn-counterparts follow. These four derivatives reactivated cyclosarin-inhibited $\mathrm{BChE}$ up to $70 \%$ in two hours of reactivation. Although the maximal percentage of reactivation was similar to the standard oxime HI- 6 , the observed reactivation rate was about four-fold slower than that obtained for HI-6. BChE inhibited by other nerve agents was resistant to reactivation with these compounds.

Nevertheless, oximes with 4-methoxyphenyl and 4-chlorophenyl substituents 14 and 15 seem to be successful in the reactivation of cyclosarin-inhibited human BChE. These findings are in accordance with our recent studies with quinuclidine-3 oximes from which the most potent reactivators of cyclosarin-inhibited BChE were bromo- or chlorobenzyl oximes [24]. 


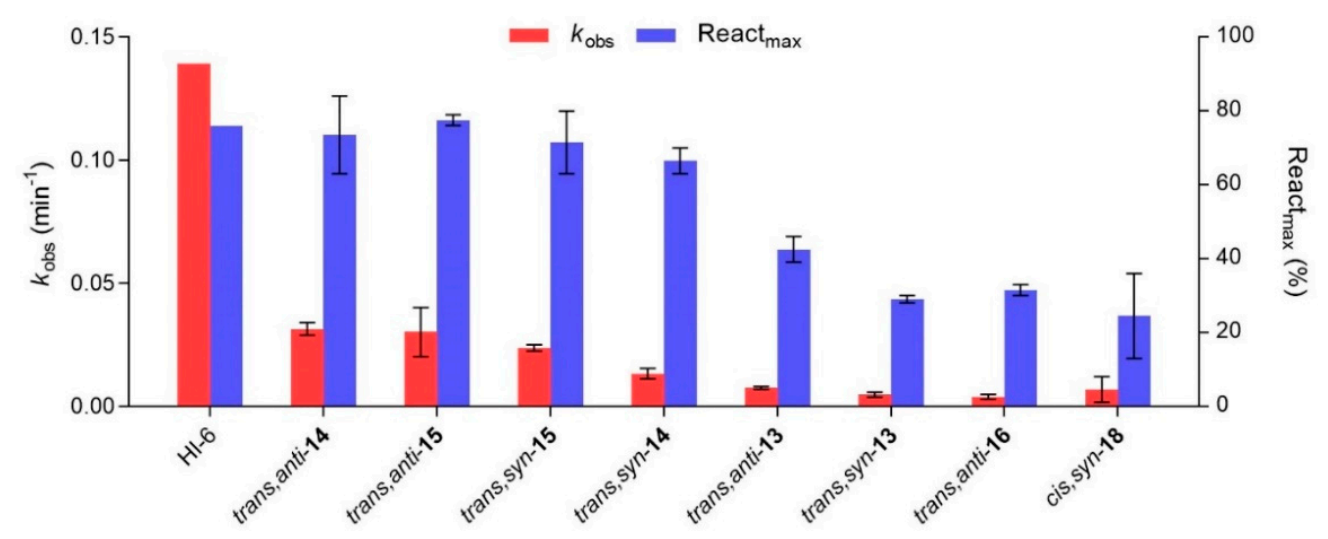

Figure 4. Reactivation of cyclosarin-inhibited human BChE with eight thienostilbene oxime derivatives and standard oxime HI-6 (0.1 mM) given as observed reactivation constant $\left(k_{\text {obs }}\right)$ and maximal reactivation in $22 \mathrm{~h}$.

\subsection{Docking of Potential Reactivators}

To gain insight into the structure of the enzyme-inhibitor complex after the oxime approaches the active site, we performed docking of thienostilbene oximes into inhibited cholinesterase. According to our experimental results, the significant reactivation ability of tested thienostilbene oximes is found only for cyclosarin-inhibited $\mathrm{BChE}$; therefore, we conducted docking of the compounds of interest into the active site of $\mathrm{BChE}$ inhibited by cyclosarin. It should be mentioned that, unlike the cyclosarin-inhibited $\mathrm{AChE}$, the crystal structure of cyclosarin-inhibited $\mathrm{BChE}$ is not available in the literature. To solve this obstacle, we tried to use the conformation of cyclosarin bound to AChE (from PDB structure 3ZLU [25]) and incorporate it within the active site of BChE. However, because of the differences between active sites of $\mathrm{BChE}$ and $\mathrm{AChE}$, specifically, due to the positioning of His438 in BChE, this approach resulted in sterically unfavorable interactions (Figure S1). Cuya et al. performed a molecular dynamics study [26] to obtain the most realistic inhibitor conformation, but they also used cyclosarin-inhibited AChE. Therefore, in our work, the experimentally obtained crystal structure of $\mathrm{BChE}$ inhibited by tabun is utilized, replacing the dimethylamino and ethoxy groups of tabun with methyl and cyclohexyloxy groups of cyclosarin, respectively. The orientation of replaced substituents in the resulting cyclosarin conformation was as similar to the structure in $\mathrm{AChE}$ as possible, given the steric limitations in the active site of $\mathrm{BChE}$ (Figure S2).

As the compounds with the best reactivating capability, trans,anti-14 and trans,anti-15 were chosen for docking into the active site of cyclosarin-inhibited BChE. For the sake of comparison, we also performed docking of the poor reactivator cis,syn-18. The structures of the active site of cyclosarin-inhibited $\mathrm{BChE}$ with docked thienostilbene oximes are shown in Figure 5. For each of these three oximes, all conformations obtained by docking into the inhibited active site are superimposed, so the most abundant orientation of the reactivator is clearly depicted.

Molecules 14 and 15 take various conformations, but it is visible from the presentations in Figure 5 that, in most cases, the oxime group is oriented toward His438. In contrast, for the molecule cis,syn-18, its oxime group is always oriented in the opposite direction. The orientation of the oxime group is relevant for the reactivation, as well as its proximity to His 438 and the phosphorus atom of cyclosarin, because the first step of reactivation is a nucleophilic attack of the oxygen of the oxime group to phosphorus. Since the oximes in this study entered the active site in neutral form, the first step of efficient reactivation should be preceded by the total (or at least partial) deprotonation of the oxime group. This deprotonation is possible only if the proton of the oxime is in a sterically favorable position to the unprotonated nitrogen atom of His438. Although neutral histidine is a weak base and thus unable to deprotonate the oxime group, here it is placed close to Glu325, which accepts the proton of the epsilon nitrogen and thus facilitates proton transfer from the oxime to 
the delta nitrogen of histidine. Figure 5 shows that none of the conformations obtained by docking of cis,syn-18 are not in a favorable position to proceed with reactivation. In contrast, for $\mathbf{1 4}$ and 15, most conformers are placed so as to enable reactivation. In Figure 6, the most favorable conformations of docked molecules $\mathbf{1 4}$ and $\mathbf{1 5}$ are presented, along with some geometry parameters.
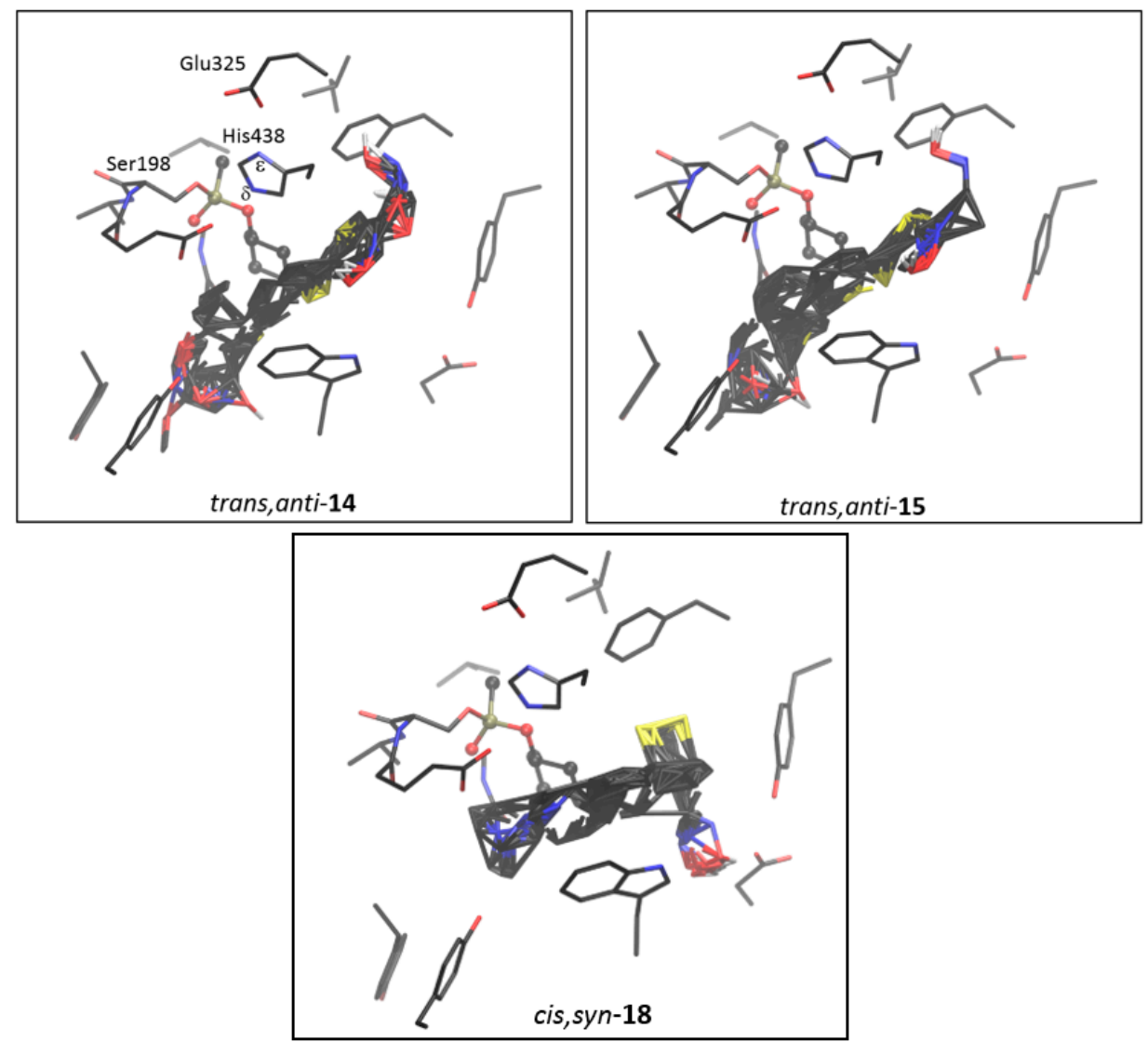

Figure 5. Structures of the active site of cyclosarin-inhibited BChE with docked thienostilbene oximes: trans,anti-14, trans,anti-15, and cis,syn-18. Cyclosarin (presented using the ball and stick model) is covalently bound to Ser198.
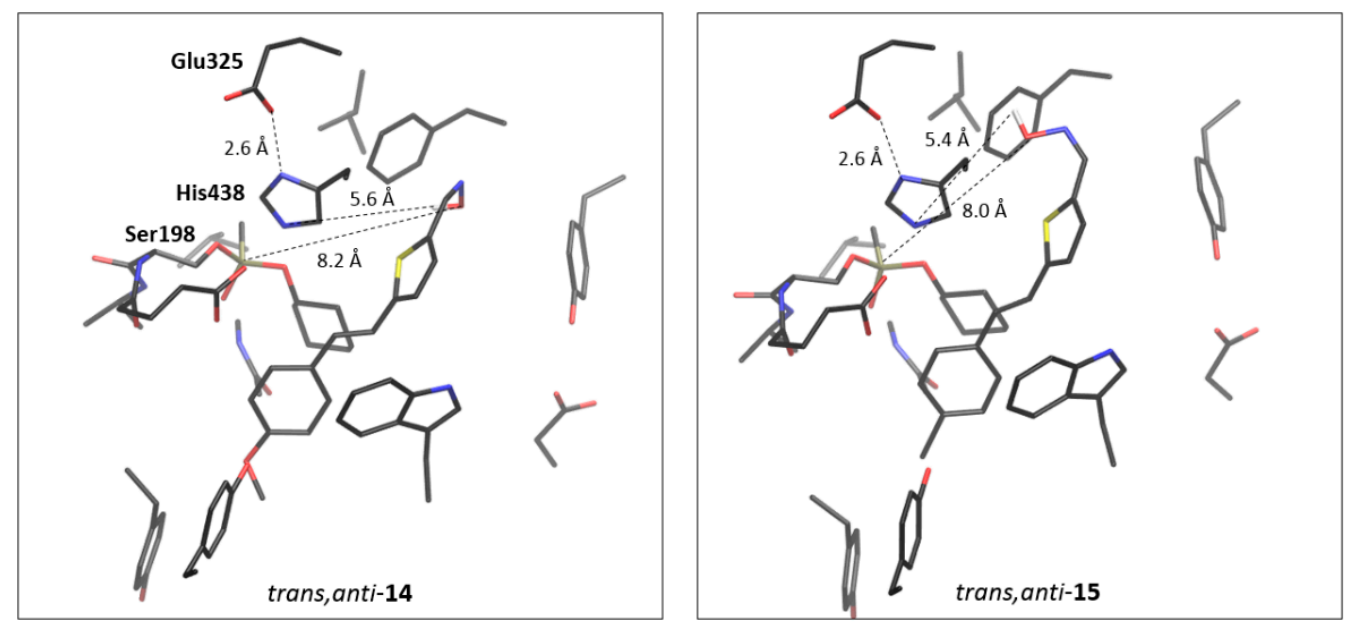

Figure 6. The most favorable conformation of trans,anti-14 and trans, anti-15 docked into the active site of cyclosarin-inhibited BChE. 
Figure 6 shows that the structures of the complexes formed between trans,anti-14 and trans,anti-15 with the active site of cyclosarin-inhibited BChE are favorable for the reactivation of the enzyme. As already mentioned, the thienostilbene oximes studied here are not in ionic form; this is important because neutral oximes have an improved ability to cross the blood-brain barrier compared to charged ones. However, to be able to break the covalent P-O bond between the inhibitor and catalytic Ser198, the oxime should be totally or partially deprotonated by histidine, such as in the semi-empirical calculations of $\mathrm{BChE}$ reactivation reported recently [27]. Therefore, the proximity of the oxime to histidine along with the position of the oxime group, which provides the absence of steric hindrance for the oxygen attack of phosphorus, are crucial for efficient reactivation.

In the static structure obtained by docking, the distance between the covalently bound inhibitor and oxygen of the neutral oxime is too big to enable the reaction to start immediately (the nucleophilic attack of oxygen to phosphorus). However, the favorable orientation of oxime and its proximity to His 438 make this process feasible. To additionally scrutinize this conclusion, we performed scanning of the Potential Energy Surface (PES), utilizing quantum chemistry calculations (see details in section Materials and Methods) on a small-model system consisting of His448, Glu325, and Ser198 with covalently bound cyclosarin and thienostilbene oxime trans,anti-15. Scanning the PES involved crude geometry optimization for the set of structures obtained by an incremental $(0.1 \AA)$ decrease in the distance between the oxygen of oxime and phosphorus, $d\left(\mathrm{O}_{\text {Oxime }}-\mathrm{P}\right)$. The starting value of the distance was $8 \AA$. The system's total energy also decreased until the oxygen approached phosphorus at $d=4.3 \AA$ (Figure S3 and Table S1). Further shortening the $d\left(\mathrm{O}_{\text {Oxime }}-\mathrm{P}\right)$ did not cause significant energy change until the oxygen was $3 \AA$ from phosphorus. It may be concluded that the approximate equilibria distance $d\left(\mathrm{O}_{\text {Oxime }}-\mathrm{P}\right)$ before the start of reaction between oxime and cyclosarin is between 4 and $3 \AA$. It is important to emphasize that this structure may be achieved spontaneously (the process being exothermic, and more relevant, without energy barriers), starting from the first geometry obtained by docking.

The investigation performed in this section does not aim to answer how different substituents at the phenyl unit of thienostilbene oximes affect their reactivating ability. However, the presented results indicate that trans, anti-thienostilbene oximes have a conformational advantage compared to their cis,syn-counterparts, thus rationalizing the experimental findings shown in Figure 4.

\section{Materials and Methods}

\subsection{General}

Petroleum ether (PE), bp $40-60^{\circ} \mathrm{C}$, was used. Solvents were purified by distillation. Column chromatography was carried out on columns with silica gel (Fluka 0.063-0.2 nm and Fluka $60 \AA$, technical grade). TLC was carried out using plates coated with silica gel $\left(0.2 \mathrm{~mm}\right.$, Kiselgel $\left.60 \mathrm{~F}_{254}\right)$. Organic layers were routinely dried with anhydrous $\mathrm{MgSO}_{4}$ and evaporated using a rotary evaporator. ${ }^{1} \mathrm{H}$ and ${ }^{13} \mathrm{C}$ NMR spectra were recorded on a spectrometer at $600 \mathrm{MHz}$. All NMR spectra were measured in $\mathrm{CDCl}_{3}$ using tetramethylsilane as a reference. The following abbreviations were used in the NMR spectra: $\mathrm{s}$ (singlet), $\mathrm{d}$ (doublet), $\mathrm{t}$ (triplet), $\mathrm{q}$ (quartet), dd (doublet of doublets), and $\mathrm{m}$ (multiplet). For the full characterization of targeted oximes, the additional techniques 2D-CH correlation (HSQC) and 2D-HH-COSY were used. Mass spectra were obtained on a UPLC-MS system. Melting points were obtained using a microscope-equipped apparatus and have not been corrected. HRMS analyses were carried out on a mass spectrometer (MALDI TOF/TOF analyzer), equipped with an Nd:YAG laser operating at $355 \mathrm{~nm}$ with a firing rate of $200 \mathrm{~Hz}$ in the positive $(\mathrm{H}+)$ or negative $(-\mathrm{H})$ ion reflector mode. The initial phosphonium salts were prepared in the laboratory from the corresponding bromides, while the other starting compounds used were purchased chemicals. 


\subsection{Synthesis of Thienostilbenes 1-6}

Thienostilbenes 1-6 were prepared by the Wittig reaction. The corresponding phosphonium salt (11 mmol) was dissolved in $130 \mathrm{~mL}$ of EtOH (dried on $4 \AA$ sieves) in a three-necked flask. The appropriate aldehyde $(10 \mathrm{mmol})$ was added. The content of the flask was mixed on a magnetic stirrer and purged with nitrogen. The reaction mixture was heated to $40^{\circ} \mathrm{C}$ for $30 \mathrm{~min}$. The NaOEt obtained from $\mathrm{Na}(11 \mathrm{mmol})$ reacted with dry $\mathrm{EtOH}$ $(10 \mathrm{~mL})$ and was added dropwise to the flask. Upon completion of the dropwise addition, the system was closed, and the reaction mixture was stirred on a magnetic stirrer overnight at room temperature. $\mathrm{EtOH}$ was applied to a rotary evaporator under reduced pressure. Extraction was carried out in a system of toluene and water, after which the organic layer was dried over $\mathrm{MgSO}_{4}$, filtered, and the toluene was evaporated. The dry reaction mixture was purified by column chromatography on silica gel using a PE/E or PE/DCM variable polarity eluent. The column chromatography yielded mixtures of cis- and trans-isomers of the desired products 1-6, which were used as reactants in further Vilsmeier formylation reactions. The spectroscopic data for the geometrical isomers of compounds 1-6 were obtained from the ${ }^{1} \mathrm{H}$ NMR spectra of the mixtures.<smiles>Cc1ccc(C)cc1</smiles>

cis-1

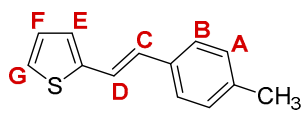

trans-1

cis-2-(4-methylstyryl)thiophene (cis-1) [28]: $404 \mathrm{mg}(18.4 \%) ; R_{f}$ (petroleum ether) $=0.71$; ${ }^{1} \mathrm{H}$ NMR $\left(\mathrm{CDCl}_{3}, 600 \mathrm{MHz}\right) \delta / \mathrm{ppm}: 7.14-7.21\left(\mathrm{~m}, 4 \mathrm{H}, \mathrm{H}_{\mathrm{A}}, \mathrm{H}_{\mathrm{B}}\right), 7.09(\mathrm{~d}, 1 \mathrm{H}, J=4.7 \mathrm{~Hz}$, $\left.\mathrm{H}_{\mathrm{G}}\right), 6.98\left(\mathrm{~d}, 1 \mathrm{H}, J=3.6 \mathrm{~Hz}, \mathrm{H}_{\mathrm{E}}\right), 6.89\left(\mathrm{dd}, 1 \mathrm{H}, J=4.7 ; 3.6 \mathrm{~Hz}, \mathrm{H}_{\mathrm{F}}\right), 6.66(\mathrm{~d}, 1 \mathrm{H}, J=12.1 \mathrm{~Hz}$, $\left.\mathrm{H}_{\mathrm{C} / \mathrm{D}}\right), 6.54\left(\mathrm{~d}, 1 \mathrm{H}, J=12.1 \mathrm{~Hz}, \mathrm{H}_{\mathrm{C} / \mathrm{D}}\right), 2.37\left(\mathrm{~s}, 3 \mathrm{H},-\mathrm{CH}_{3}\right)$;

trans-2-(4-methylstyryl)thiophene (trans-1) [28,29]: $810 \mathrm{mg}(36.8 \%) ; R_{f}$ (petroleum ether) $=0.57 ;{ }^{1} \mathrm{H} \mathrm{NMR}\left(\mathrm{CDCl}_{3}, 600 \mathrm{MHz}\right) \delta / \mathrm{ppm}: 7.37\left(\mathrm{~d}, 2 \mathrm{H}, J=7.8 \mathrm{~Hz}, \mathrm{H}_{\mathrm{A} / \mathrm{B}}\right), 7.26(\mathrm{~d}, 1 \mathrm{H}$, $\left.J=5.2 \mathrm{~Hz}, \mathrm{H}_{\mathrm{G}}\right), 7.21-7.14\left(\mathrm{~m}, 3 \mathrm{H}, \mathrm{H}_{\mathrm{C} / \mathrm{D}}, \mathrm{H}_{\mathrm{A} / \mathrm{B}}\right), 7.05\left(\mathrm{~d}, 1 \mathrm{H}, J=3.6 \mathrm{~Hz}, \mathrm{H}_{\mathrm{E}}\right), 7.00(\mathrm{dd}, 1 \mathrm{H}$, $\left.J=5.2 ; 3.6 \mathrm{~Hz}, \mathrm{H}_{\mathrm{F}}\right), 6.91\left(\mathrm{~d}, 1 \mathrm{H}, J=16.0 \mathrm{~Hz}, \mathrm{H}_{\mathrm{C} / \mathrm{D}}\right), 2.36\left(\mathrm{~s}, 3 \mathrm{H},-\mathrm{CH}_{3}\right)$;

MS (ESI) $m / z$ (\%, fragment): $201\left(100, \mathrm{M}+\mathrm{H}^{+}\right)$; HRMS $(\mathrm{m} / z)$ for $\mathrm{C}_{13} \mathrm{H}_{12} \mathrm{~S}$ (obtained for the pure mixture of geometrical isomers): $[\mathrm{M}+\mathrm{H}]^{+}$calcd $=201.0659,[\mathrm{M}+\mathrm{H}]^{+}$measured $=201.0660$.<smiles>COc1ccc(/C=C/c2sccc2F)cc1</smiles>

cis-2<smiles>COc1ccc(/C=C/c2sccc2F)cc1</smiles>

trans-2

cis-2-(4-methoxystyryl)thiophene (cis-2) [30]: $403 \mathrm{mg}$ (17.0\%); $R_{f}$ (petroleum ether/ diethyl ether $(1 \%))=0.30 ;{ }^{1} \mathrm{H}$ NMR $\left(\mathrm{CDCl}_{3}, 600 \mathrm{MHz}\right) \delta / \mathrm{ppm}: 7.29(\mathrm{~d}, 2 \mathrm{H}, J=8.7 \mathrm{~Hz}$, $\left.\mathrm{H}_{\mathrm{A} / \mathrm{B}}\right), 7.09\left(\mathrm{~d}, 1 \mathrm{H}, J=4.9 \mathrm{~Hz}, \mathrm{H}_{\mathrm{G}}\right), 6.98\left(\mathrm{dd}, 1 \mathrm{H}, J=4.9 ; 3.6 \mathrm{~Hz}, \mathrm{H}_{\mathrm{F}}\right), 6.98(\mathrm{~d}, 1 \mathrm{H}, J=3.6 \mathrm{~Hz}$, $\left.\mathrm{H}_{\mathrm{E}}\right), 6.87\left(\mathrm{~d}, 2 \mathrm{H}, J=8.7 \mathrm{~Hz}, \mathrm{H}_{\mathrm{A} / \mathrm{B}}\right), 6.63\left(\mathrm{~d}, 1 \mathrm{H}, J=11.8 \mathrm{~Hz}, \mathrm{H}_{\mathrm{C} / \mathrm{D}}\right), 6.51(\mathrm{~d}, 1 \mathrm{H}, J=11.8 \mathrm{~Hz}$, $\left.\mathrm{H}_{\mathrm{C} / \mathrm{D}}\right), 3.83\left(\mathrm{~s}, 3 \mathrm{H},-\mathrm{OCH}_{3}\right)$;

trans-2-(4-methoxystyryl)thiophene (trans-2) [29]: $803 \mathrm{mg}$ (33.8\%); $R_{f}$ (petroleum ether/ diethyl ether $(1 \%))=0.23 ;{ }^{1} \mathrm{H}$ NMR $\left(\mathrm{CDCl}_{3}, 600 \mathrm{MHz}\right) \delta / \mathrm{ppm}: 7.40(\mathrm{~d}, 2 \mathrm{H}, J=8.4 \mathrm{~Hz}$, $\left.\mathrm{H}_{\mathrm{A} / \mathrm{B}}\right), 7.15\left(\mathrm{~d}, 1 \mathrm{H}, J=4.9 \mathrm{~Hz}, \mathrm{H}_{\mathrm{G}}\right), 7.10\left(\mathrm{~d}, 1 \mathrm{H}, J=15.9 \mathrm{~Hz}, \mathrm{H}_{\mathrm{C} / \mathrm{D}}\right), 7.02(\mathrm{~d}, 1 \mathrm{H}, J=3.6 \mathrm{~Hz}$, $\left.\mathrm{H}_{\mathrm{E}}\right), 7.01\left(\mathrm{~d}, 1 \mathrm{H}, J=15.9 \mathrm{~Hz}, \mathrm{H}_{\mathrm{C} / \mathrm{D}}\right), 6.89\left(\mathrm{~d}, 2 \mathrm{H}, J=8.4 \mathrm{~Hz}, \mathrm{H}_{\mathrm{A} / \mathrm{B}}\right), 6.88(\mathrm{dd}, 1 \mathrm{H}, J=4.9$; $\left.3.6 \mathrm{~Hz}, \mathrm{H}_{\mathrm{F}}\right), 3.83\left(\mathrm{~s}, 3 \mathrm{H},-\mathrm{OCH}_{3}\right)$;

MS (ESI) $m / z\left(\%\right.$, fragment): $217\left(100, \mathrm{M}+\mathrm{H}^{+}\right)$; HRMS $(\mathrm{m} / z)$ for $\mathrm{C}_{13} \mathrm{H}_{12} \mathrm{OS}$ (obtained for the pure mixture of geometrical isomers): $[\mathrm{M}+\mathrm{H}]^{+}$calcd $=217.0609,[\mathrm{M}+\mathrm{H}]^{+}$measured $=217.0605$. 


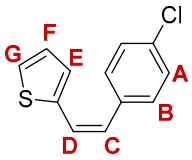

cis-3

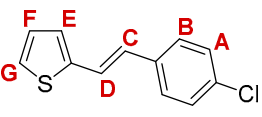

trans-3

cis-2-(4-chlorostyryl)thiophene (cis-3): $591 \mathrm{mg}(24.4 \%) ; R_{f}$ (petroleum ether) $=0.75 ;{ }^{1} \mathrm{H}$ $\operatorname{NMR}\left(\mathrm{CDCl}_{3}, 600 \mathrm{MHz}\right) \delta / \mathrm{ppm}$ : 7.33-7.28 (m, 4H, $\left.\mathrm{H}_{\mathrm{A} / \mathrm{B}}\right), 7.12\left(\mathrm{~d}, 1 \mathrm{H}, J=5.0 \mathrm{~Hz}, \mathrm{H}_{\mathrm{G}}\right), 6.97$ $\left(\mathrm{d}, 1 \mathrm{H}, J=3.6 \mathrm{~Hz}, \mathrm{H}_{\mathrm{E}}\right), 6.90\left(\mathrm{dd}, 1 \mathrm{H}, J=5.0 ; 3.6 \mathrm{~Hz}, \mathrm{H}_{\mathrm{F}}\right), 6.71\left(\mathrm{~d}, 1 \mathrm{H}, J=11.8 \mathrm{~Hz}, \mathrm{H}_{\mathrm{C} / \mathrm{D}}\right)$, $6.50\left(\mathrm{~d}, 1 \mathrm{H}, J=11.8 \mathrm{~Hz}, \mathrm{H}_{\mathrm{C} / \mathrm{D}}\right)$;

trans-2-(4-chlorostyryl)thiophene (trans-3) [29]: $629 \mathrm{mg}(26.0 \%) ; R_{f}$ (petroleum ether) = $0.65 ;{ }^{1} \mathrm{H} \mathrm{NMR}\left(\mathrm{CDCl}_{3}, 600 \mathrm{MHz}\right) \delta / \mathrm{ppm}: 7.39\left(\mathrm{~d}, 2 \mathrm{H}, J=8.5 \mathrm{~Hz}, \mathrm{H}_{\mathrm{A} / \mathrm{B}}\right), 7.33-7.28(\mathrm{~m}, 2 \mathrm{H}$, $\left.\mathrm{H}_{\mathrm{A} / \mathrm{B}}\right), 7.21\left(\mathrm{~d}, 1 \mathrm{H}, J=5.0 \mathrm{~Hz}, \mathrm{H}_{\mathrm{G}}\right), 7.20\left(\mathrm{~d}, 1 \mathrm{H}, J=16.2 \mathrm{~Hz}, \mathrm{H}_{\mathrm{C} / \mathrm{D}}\right), 7.08(\mathrm{~d}, 1 \mathrm{H}, J=3.4 \mathrm{~Hz}$, $\left.\mathrm{H}_{\mathrm{E}}\right), 7.01\left(\mathrm{dd}, 1 \mathrm{H}, J=5.0 ; 3.4 \mathrm{~Hz}, \mathrm{H}_{\mathrm{F}}\right), 6.87\left(\mathrm{~d}, 1 \mathrm{H}, J=16.2 \mathrm{~Hz}, \mathrm{H}_{\mathrm{C} / \mathrm{D}}\right)$;

MS (ESI) $m / z$ (\%, fragment): 221/223 (80, $\left.\mathrm{M}+\mathrm{H}^{+}\right), 186(100)$; HRMS $(m / z)$ for $\mathrm{C}_{12} \mathrm{H}_{9} \mathrm{ClS}$ (obtained for the pure mixture of geometrical isomers): $[\mathrm{M}+\mathrm{H}]^{+}$calcd $=221.0112,[\mathrm{M}+\mathrm{H}]^{+}$measured $=221.0114$.

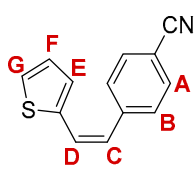

cis-4

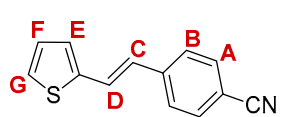

trans-4

cis-4-(2-(thiophen-2-yl)vinyl)benzonitrile (cis-4): $831 \mathrm{mg}$ (35.8\%); $R_{f}$ (petroleum ether/ diethyl ether $(30 \%))=0.52 ;{ }^{1} \mathrm{H}$ NMR $\left(\mathrm{CDCl}_{3}, 600 \mathrm{MHz}\right) \delta / \mathrm{ppm}: 7.63(\mathrm{~d}, 2 \mathrm{H}, J=8.0 \mathrm{~Hz}$, $\left.\mathrm{H}_{\mathrm{A} / \mathrm{B}}\right), 7.47\left(\mathrm{~d}, 2 \mathrm{H}, J=8.0 \mathrm{~Hz}, \mathrm{H}_{\mathrm{A} / \mathrm{B}}\right), 7.14\left(\mathrm{~d}, 1 \mathrm{H}, J=4.9 \mathrm{~Hz}, \mathrm{H}_{\mathrm{G}}\right), 6.98\left(\mathrm{~d}, 1 \mathrm{H}, J=3.6 \mathrm{~Hz}, \mathrm{H}_{\mathrm{E}}\right)$, $6.92\left(\mathrm{dd}, 1 \mathrm{H}, J=4.9 ; 3.6 \mathrm{~Hz}, \mathrm{H}_{\mathrm{F}}\right), 6.79\left(\mathrm{~d}, 1 \mathrm{H}, J=12.0 \mathrm{~Hz}, \mathrm{H}_{\mathrm{C} / \mathrm{D}}\right), 6.52(\mathrm{~d}, 1 \mathrm{H}, J=12.0 \mathrm{~Hz}$, $\mathrm{H}_{\mathrm{C} / \mathrm{D})}$;

trans-4-(2-(thiophen-2-yl)vinyl)benzonitrile (trans-4) [29]: $585 \mathrm{mg}(25.2 \%) ; R_{f}$ (petroleum ether/diethyl ether $(30 \%))=0.43 ;{ }^{1} \mathrm{H}$ NMR $\left(\mathrm{CDCl}_{3}, 600 \mathrm{MHz}\right) \delta / \mathrm{ppm}: 7.62(\mathrm{~d}, 2 \mathrm{H}, J=8.3 \mathrm{~Hz}$, $\left.\mathrm{H}_{\mathrm{A} / \mathrm{B}}\right), 7.53\left(\mathrm{~d}, 2 \mathrm{H}, J=8.3 \mathrm{~Hz}, \mathrm{H}_{\mathrm{A} / \mathrm{B}}\right), 7.34\left(\mathrm{~d}, 1 \mathrm{H}, J=16.2 \mathrm{~Hz}, \mathrm{H}_{\mathrm{C} / \mathrm{D}}\right), 7.28(\mathrm{~d}, 1 \mathrm{H}, J=5.1 \mathrm{~Hz}$, $\left.\mathrm{H}_{\mathrm{G}}\right), 7.15\left(\mathrm{~d}, 1 \mathrm{H}, J=3.5 \mathrm{~Hz}, \mathrm{H}_{\mathrm{E}}\right), 7.04\left(\mathrm{dd}, 1 \mathrm{H}, J=5.1 ; 3.5 \mathrm{~Hz}, \mathrm{H}_{\mathrm{F}}\right), 6.90(\mathrm{~d}, 1 \mathrm{H}, J=16.2 \mathrm{~Hz}$, $\mathrm{H}_{\mathrm{C} / \mathrm{D})}$;

MS (ESI) $m / z\left(\%\right.$, fragment): $212\left(100, \mathrm{M}+\mathrm{H}^{+}\right)$; HRMS $(m / z)$ for $\mathrm{C}_{13} \mathrm{H}_{9} \mathrm{NS}$ (obtained for the pure mixture of geometrical isomers): $[\mathrm{M}+\mathrm{H}]^{+}$calcd $=212.0458,[\mathrm{M}+\mathrm{H}]^{+}$measured $=212.0456$.<smiles>O=[N+]([O-])c1ccc(-c2cccs2)cc1</smiles>

cis-5<smiles>O=[N+]([O-])c1ccc(/C=C/c2ccc[se]2)cc1</smiles>

trans-5

cis-2-(4-nitrostyryl)thiophene (cis-5): $582 \mathrm{mg}$ (22.9\%); $R_{f}$ (petroleum ether/diethyl ether $(15 \%))=0.63 ;{ }^{1} \mathrm{H}$ NMR $\left(\mathrm{CDCl}_{3}, 600 \mathrm{MHz}\right) \delta / \mathrm{ppm}: 8.19\left(\mathrm{~d}, 2 \mathrm{H}, J=8.4 \mathrm{~Hz}, \mathrm{H}_{\mathrm{A} / \mathrm{B}}\right), 7.53(\mathrm{~d}$, $\left.2 \mathrm{H}, J=8.4 \mathrm{~Hz}, \mathrm{H}_{\mathrm{A} / \mathrm{B}}\right), 7.17\left(\mathrm{~d}, 1 \mathrm{H}, J=5.2 \mathrm{~Hz}, \mathrm{H}_{\mathrm{G}}\right), 6.99\left(\mathrm{~d}, 1 \mathrm{H}, J=3.5 \mathrm{~Hz}, \mathrm{H}_{\mathrm{E}}\right), 6.99(\mathrm{dd}, 1 \mathrm{H}$, $\left.J=5.2 ; 3.5 \mathrm{~Hz}, \mathrm{H}_{\mathrm{F}}\right), 6.83\left(\mathrm{~d}, 1 \mathrm{H}, J=12.0 \mathrm{~Hz}, \mathrm{H}_{\mathrm{C} / \mathrm{D}}\right), 6.55\left(\mathrm{~d}, 1 \mathrm{H}, J=12.0 \mathrm{~Hz}, \mathrm{H}_{\mathrm{C} / \mathrm{D}}\right)$;

trans-2-(4-nitrostyryl)thiophene (trans-5) [29]: $630 \mathrm{mg}$ (24.8\%); $R_{f}$ (petroleum ether/ diethyl ether $(15 \%))=0.51 ;{ }^{1} \mathrm{H} \mathrm{NMR}\left(\mathrm{CDCl}_{3}, 600 \mathrm{MHz}\right) \delta / \mathrm{ppm}: 8.21(\mathrm{~d}, 2 \mathrm{H}, J=8.6 \mathrm{~Hz}$, $\left.\mathrm{H}_{\mathrm{A} / \mathrm{B}}\right), 7.58\left(\mathrm{~d}, 2 \mathrm{H}, J=8.6 \mathrm{~Hz}, \mathrm{H}_{\mathrm{A} / \mathrm{B}}\right), 7.39\left(\mathrm{~d}, 1 \mathrm{H}, J=16.1 \mathrm{~Hz}, \mathrm{H}_{\mathrm{C} / \mathrm{D}}\right), 7.30(\mathrm{~d}, 1 \mathrm{H}, J=5.2 \mathrm{~Hz}$, $\left.\mathrm{H}_{\mathrm{G}}\right), 7.18\left(\mathrm{~d}, 1 \mathrm{H}, J=3.7 \mathrm{~Hz}, \mathrm{H}_{\mathrm{E}}\right), 7.05\left(\mathrm{dd}, 1 \mathrm{H}, J=5.2 ; 3.7 \mathrm{~Hz}, \mathrm{H}_{\mathrm{F}}\right), 6.95(\mathrm{~d}, 1 \mathrm{H}, J=16.1 \mathrm{~Hz}$, $\mathrm{H}_{\mathrm{C} / \mathrm{D}}$ ); 
MS (ESI) $m / z$ (\%, fragment): $232\left(100, \mathrm{M}+\mathrm{H}^{+}\right)$; $\mathrm{HRMS}(\mathrm{m} / z)$ for $\mathrm{C}_{12} \mathrm{H}_{9} \mathrm{NO}_{2} \mathrm{~S}$ (obtained for the pure mixture of geometrical isomers): $[\mathrm{M}+\mathrm{H}]^{+}{ }_{\text {calcd }}=232.0356,[\mathrm{M}+\mathrm{H}]^{+}$measured $=232.0354$.<smiles></smiles>

cis-6



trans-6

cis- $N, N$-dimethyl-4-(2-(thiophen-2-yl)vinyl)aniline (cis-6) [31]: $524 \mathrm{mg}(20.8 \%) ; R_{f}$ (petroleum ether/diethyl ether $(30 \%))=0.55 ;{ }^{1} \mathrm{H} \mathrm{NMR}\left(\mathrm{CDCl}_{3}, 600 \mathrm{MHz}\right) \delta / \mathrm{ppm}: 7.28(\mathrm{~d}$, $\left.2 \mathrm{H}, J=8.7 \mathrm{~Hz}, \mathrm{H}_{\mathrm{A} / \mathrm{B}}\right), 7.10\left(\mathrm{~d}, 1 \mathrm{H}, J=5.2 \mathrm{~Hz}, \mathrm{H}_{\mathrm{G}}\right), 7.02\left(\mathrm{~d}, 1 \mathrm{H}, J=3.6 \mathrm{~Hz}, \mathrm{H}_{\mathrm{E}}\right), 6.91(\mathrm{dd}, 1 \mathrm{H}$, $\left.J=5.2 ; 3.6 \mathrm{~Hz}, \mathrm{H}_{\mathrm{F}}\right), 6.69\left(\mathrm{~d}, 2 \mathrm{H}, J=8.7 \mathrm{~Hz}, \mathrm{H}_{\mathrm{A} / \mathrm{B}}\right), 6.54\left(\mathrm{~d}, 1 \mathrm{H}, J=11.9 \mathrm{~Hz}, \mathrm{H}_{\mathrm{C} / \mathrm{D}}\right), 6.49(\mathrm{~d}$, $\left.1 \mathrm{H}, J=11.9 \mathrm{~Hz}, \mathrm{H}_{\mathrm{C} / \mathrm{D}}\right), 2.98\left(\mathrm{~s}, 6 \mathrm{H},-\mathrm{N}\left(\mathrm{CH}_{3}\right)_{2}\right)$;

trans-N,N-dimethyl-4-(2-(thiophen-2-yl)vinyl)aniline (trans-6) [31]: $630 \mathrm{mg}(25.0 \%) ; R_{f}$ (petroleum ether/diethyl ether $(30 \%))=0.47 ;{ }^{1} \mathrm{H} \mathrm{NMR}\left(\mathrm{CDCl}_{3}, 600 \mathrm{MHz}\right) \delta / \mathrm{ppm}: 7.37(\mathrm{~d}$, $\left.2 \mathrm{H}, J=8.7 \mathrm{~Hz}, \mathrm{H}_{\mathrm{A} / \mathrm{B}}\right), 7.12\left(\mathrm{~d}, 1 \mathrm{H}, J=4.7 \mathrm{~Hz}, \mathrm{H}_{\mathrm{G}}\right), 7.05\left(\mathrm{~d}, 1 \mathrm{H}, J=16.0 \mathrm{~Hz}, \mathrm{H}_{\mathrm{C} / \mathrm{D}}\right), 6.99(\mathrm{~d}$, $\left.1 \mathrm{H}, J=3.6 \mathrm{~Hz}, \mathrm{H}_{\mathrm{E}}\right), 6.98\left(\mathrm{dd}, 1 \mathrm{H}, J=4.7 ; 3.6 \mathrm{~Hz}, \mathrm{H}_{\mathrm{F}}\right), 6.88\left(\mathrm{~d}, 1 \mathrm{H}, J=16.0 \mathrm{~Hz}, \mathrm{H}_{\mathrm{C} / \mathrm{D}}\right), 6.71$ $\left(\mathrm{d}, 2 \mathrm{H}, J=8.7 \mathrm{~Hz}, \mathrm{H}_{\mathrm{A} / \mathrm{B}}\right), 2.99\left(\mathrm{~s}, 6 \mathrm{H},-\mathrm{N}\left(\mathrm{CH}_{3}\right)_{2}\right)$;

MS (ESI) $m / z$ (\%, fragment): $230\left(100, \mathrm{M}+\mathrm{H}^{+}\right)$; $\mathrm{HRMS}(\mathrm{m} / \mathrm{z})$ for $\mathrm{C}_{14} \mathrm{H}_{15} \mathrm{NS}$ (obtained for the pure mixture of geometrical isomers): $[\mathrm{M}+\mathrm{H}]^{+}{ }_{\text {calcd }}=230.0929,[\mathrm{M}+\mathrm{H}]^{+}$measured $=230.0925$.

\subsection{Synthesis of Aldehydes 7-12}

The obtained thienostilbenes, products of Wittig reactions 1-6, were subjected to Vilsmeier formylation. The selected thienostilbenes, as a mixture of isomers, were dissolved in $2 \mathrm{~mL}$ of DMF and stirred for $10 \mathrm{~min}$ at $10{ }^{\circ} \mathrm{C}$. This temperature was achieved using a water bath with a few ice cubes and monitored with a thermometer. The weighed amount of $\mathrm{POCl}_{3}$ was slowly added dropwise. After $30 \mathrm{~min}$, the water bath was removed, and the reaction mixture was allowed to stir at room temperature. In the reactions to prepare compounds 7-9, the reaction mixture was stirred at room temperature for $48 \mathrm{~h}$. The reaction mixture for preparing compounds 10 and 11 was heated to $80{ }^{\circ} \mathrm{C}$, and the reaction mixture for preparing compound 12 to $60{ }^{\circ} \mathrm{C}$. Heating was continued for $3 \mathrm{~h}$, after which time the reaction mixture was left to stir at room temperature for $48 \mathrm{~h}$. Upon completion of the reaction, the reaction mixture was neutralized using $10 \% \mathrm{NaOH}$ solution. When neutralization was achieved, extraction was carried using diethyl ether and water. The combined organic layer was washed with water and dried over $\mathrm{MgSO}_{4}$, filtered, and the diethyl ether was evaporated on a rotary evaporator. The dry reaction mixture was purified by column chromatography on silica gel using a PE/E or PE/DCM variable polarity eluent. In the first fractions, the unreacted substrates 1-6 were isolated (mostly as cis-isomers), while in the last fractions, the desired formyl derivatives 7-12 were obtained (mostly as trans-isomers). They were further used in the preparation reactions for oximes 13-18. The spectroscopic data for the geometrical isomers were obtained from the ${ }^{1} \mathrm{H}$ NMR spectra of the mixtures of compounds 7-12.
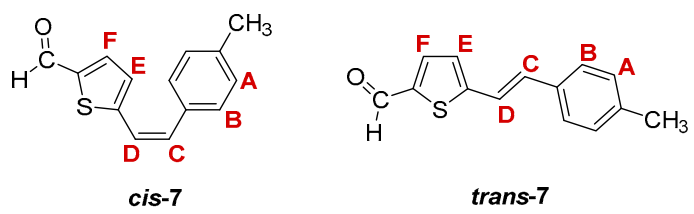

cis-5-(4-methylstyryl)thiophene-2-carbaldehyde (cis-7): $167 \mathrm{mg} \quad(12.1 \%) ; \quad R_{f}$ (petroleum ether/diethyl ether $(10 \%))=0.41 ;{ }^{1} \mathrm{H} \mathrm{NMR}\left(\mathrm{CDCl}_{3}, 600 \mathrm{MHz}\right) \delta / \mathrm{ppm}: 9.79(\mathrm{~s}$, $1 \mathrm{H},-\mathrm{CHO}), 7.36\left(\mathrm{~d}, 2 \mathrm{H}, J=8.1 \mathrm{~Hz}, \mathrm{H}_{\mathrm{A} / \mathrm{B}}\right), 7.18\left(\mathrm{~d}, 2 \mathrm{H}, J=8.1 \mathrm{~Hz}, \mathrm{H}_{\mathrm{A} / \mathrm{B}}\right), 7.18-7.12(\mathrm{~m}, 2 \mathrm{H}$, $\left.\mathrm{H}_{\mathrm{E}}, \mathrm{H}_{\mathrm{F}}\right), 6.65\left(\mathrm{~d}, 1 \mathrm{H}, J=11.7 \mathrm{~Hz}, \mathrm{H}_{\mathrm{C} / \mathrm{D}}\right), 6.54\left(\mathrm{~d}, 1 \mathrm{H}, J=11.7 \mathrm{~Hz}, \mathrm{H}_{\mathrm{C} / \mathrm{D}}\right), 2.36\left(\mathrm{~s}, 3 \mathrm{H},-\mathrm{CH}_{3}\right)$; 
trans-5-(4-methylstyryl)thiophene-2-carbaldehyde (trans-7): $335 \mathrm{mg}$ (24.2\%); $R_{f}$ (petroleum ether/diethyl ether $(10 \%))=0.27 ;{ }^{1} \mathrm{H} \mathrm{NMR}\left(\mathrm{CDCl}_{3}, 600 \mathrm{MHz}\right) \delta / \mathrm{ppm}:{ }^{1} \mathrm{H}$ $\operatorname{NMR}\left(\mathrm{CDCl}_{3}, 600 \mathrm{MHz}\right) \delta / \mathrm{ppm}: 9.85(\mathrm{~s}, 1 \mathrm{H},-\mathrm{CHO}), 7.66\left(\mathrm{~d}, 1 \mathrm{H}, J=3.8 \mathrm{~Hz}, \mathrm{H}_{\mathrm{E} / \mathrm{F}}\right), 7.40(\mathrm{~d}$, $\left.2 \mathrm{H}, J=8.9 \mathrm{~Hz}, \mathrm{H}_{\mathrm{A} / \mathrm{B}}\right), 7.14\left(\mathrm{~d}, 1 \mathrm{H}, J=16.3 \mathrm{~Hz}, \mathrm{H}_{\mathrm{C} / \mathrm{D}}\right), 7.15\left(\mathrm{~d}, 2 \mathrm{H}, J=8.9 \mathrm{~Hz}, \mathrm{H}_{\mathrm{A} / \mathrm{B}}\right), 7.13(\mathrm{~d}$, $\left.1 \mathrm{H}, J=3.8 \mathrm{~Hz}, \mathrm{H}_{\mathrm{E} / \mathrm{F}}\right), 6.90\left(\mathrm{~d}, 1 \mathrm{H}, J=16.3 \mathrm{~Hz}, \mathrm{H}_{\mathrm{C} / \mathrm{D}}\right), 2.37\left(\mathrm{~s}, 3 \mathrm{H},-\mathrm{CH}_{3}\right)$;

MS (ESI) $m / z$ (\%, fragment): $229\left(100, \mathrm{M}+\mathrm{H}^{+}\right)$; HRMS $(m / z)$ for $\mathrm{C}_{14} \mathrm{H}_{12} \mathrm{OS}$ (obtained for the pure mixture of geometrical isomers): $[\mathrm{M}+\mathrm{H}]^{+}{ }_{\text {calcd }}=229.0609,[\mathrm{M}+\mathrm{H}]^{+}$measured $=229.0608$.<smiles>COc1ccc(C=Cc2cc(F)c(C=O)s2)cc1</smiles>

cis-8

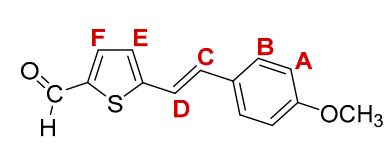

trans-8

cis-5-(4-methoxystyryl)thiophene-2-carbaldehyde (cis-8): $66 \mathrm{mg}$ (4.8\%); $R_{f}$ (petroleum ether/ diethyl ether $(10 \%))=0.20 ;{ }^{1} \mathrm{H} \mathrm{NMR}\left(\mathrm{CDCl}_{3}, 600 \mathrm{MHz}\right) \delta / \mathrm{ppm}: 9.79(\mathrm{~s}, 1 \mathrm{H},-\mathrm{C} \underline{\mathrm{HO}}), 7.55(\mathrm{~d}$, $\left.1 \mathrm{H}, J=3.9 \mathrm{~Hz}, \mathrm{H}_{\mathrm{E} / \mathrm{F}}\right), 7.45\left(\mathrm{~d}, 2 \mathrm{H}, J=8.8 \mathrm{~Hz}, \mathrm{H}_{\mathrm{A} / \mathrm{B}}\right), 7.04\left(\mathrm{~d}, 1 \mathrm{H}, J=3.9 \mathrm{~Hz}, \mathrm{H}_{\mathrm{E} / \mathrm{F}}\right), 6.87(\mathrm{~d}, 2 \mathrm{H}$, $\left.J=8.8 \mathrm{~Hz}, \mathrm{H}_{\mathrm{A} / \mathrm{B}}\right), 6.75\left(\mathrm{~d}, 1 \mathrm{H}, J=11.9 \mathrm{~Hz}, \mathrm{H}_{\mathrm{C} / \mathrm{D}}\right), 6.61\left(\mathrm{~d}, 1 \mathrm{H}, J=11.9 \mathrm{~Hz}, \mathrm{H}_{\mathrm{C} / \mathrm{D}}\right), 3.82(\mathrm{~s}, 3 \mathrm{H}$, $\left.-\mathrm{OCH}_{3}\right)$;

trans-5-(4-methoxystyryl)thiophene-2-carbaldehyde (trans-8) [32]: $261 \mathrm{mg}(19.1 \%) ; R_{f}$ (petroleum ether/diethyl ether $(10 \%))=0.15 ;{ }^{1} \mathrm{H} \mathrm{NMR}\left(\mathrm{CDCl}_{3}, 600 \mathrm{MHz}\right) \delta / \mathrm{ppm}$ : 9.83 $(\mathrm{s}, 1 \mathrm{H},-\mathrm{CHO}), 7.65\left(\mathrm{~d}, 1 \mathrm{H}, J=3.9 \mathrm{~Hz}, \mathrm{H}_{\mathrm{E} / \mathrm{F}}\right), 7.45\left(\mathrm{~d}, 2 \mathrm{H}, J=8.8 \mathrm{~Hz}, \mathrm{H}_{\mathrm{A} / \mathrm{B}}\right), 7.11(\mathrm{~d}, 1 \mathrm{H}$, $\left.J=16.1 \mathrm{~Hz}, \mathrm{H}_{\mathrm{C} / \mathrm{D}}\right), 7.10\left(\mathrm{~d}, 1 \mathrm{H}, J=3.9 \mathrm{~Hz}, \mathrm{H}_{\mathrm{E} / \mathrm{F}}\right), 7.07\left(\mathrm{~d}, 1 \mathrm{H}, J=16.1 \mathrm{~Hz}, \mathrm{H}_{\mathrm{C} / \mathrm{D}}\right), 6.91(\mathrm{~d}$, $\left.2 \mathrm{H}, J=8.8 \mathrm{~Hz}, \mathrm{H}_{\mathrm{A} / \mathrm{B}}\right), 3.84\left(\mathrm{~s}, 3 \mathrm{H},-\mathrm{OCH}_{3}\right)$;

MS (ESI) $m / z$ (\%, fragment): $245\left(100, \mathrm{M}+\mathrm{H}^{+}\right), 121(40)$; $\mathrm{HRMS}(\mathrm{m} / \mathrm{z})$ for $\mathrm{C}_{14} \mathrm{H}_{12} \mathrm{O}_{2} \mathrm{~S}$ (obtained for the pure mixture of geometrical isomers): $[\mathrm{M}+\mathrm{H}]^{+}$calcd $=245.0458,[\mathrm{M}+\mathrm{H}]^{+}{ }_{\text {measured }}=$ 245.0456.<smiles>O=Cc1ccc(-c2ccc(Cl)cc2)cc1</smiles>

cis-9

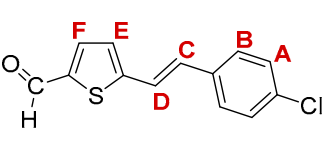

trans-9

cis-5-(4-chlorostyryl)thiophene-2-carbaldehyde (cis-9): $74 \mathrm{mg}$ (5.4\%); $R_{f}$ (petroleum ether/ diethyl ether $(30 \%))=0.56 ;{ }^{1} \mathrm{H}$ NMR $\left(\mathrm{CDCl}_{3}, 600 \mathrm{MHz}\right) \delta / \mathrm{ppm}: 9.80(\mathrm{~s}, 1 \mathrm{H},-\mathrm{CHO}), 7.56(\mathrm{~d}$, $\left.1 \mathrm{H}, J=3.9 \mathrm{~Hz}, \mathrm{H}_{\mathrm{E} / \mathrm{F}}\right), 7.34\left(\mathrm{~d}, 2 \mathrm{H}, J=8.2 \mathrm{~Hz}, \mathrm{H}_{\mathrm{A} / \mathrm{B}}\right), 7.26\left(\mathrm{~d}, 2 \mathrm{H}, J=8.2 \mathrm{~Hz}, \mathrm{H}_{\mathrm{A} / \mathrm{B}}\right), 7.02(\mathrm{~d}$, $\left.1 \mathrm{H}, J=3.9 \mathrm{~Hz}, \mathrm{H}_{\mathrm{E} / \mathrm{F}}\right), 6.74\left(\mathrm{~d}, 1 \mathrm{H}, J=12.1 \mathrm{~Hz}, \mathrm{H}_{\mathrm{C} / \mathrm{D}}\right), 6.70\left(\mathrm{~d}, 1 \mathrm{H}, J=12.1 \mathrm{~Hz}, \mathrm{H}_{\mathrm{C} / \mathrm{D}}\right)$;

trans-5-(4-chlorostyryl)thiophene-2-carbaldehyde (trans-9) [32]: $370 \mathrm{mg}$ (26.9\%); $R_{f}$ (petroleum ether/diethyl ether $(30 \%))=0.44 ;{ }^{1} \mathrm{H} \mathrm{NMR}\left(\mathrm{CDCl}_{3}, 600 \mathrm{MHz}\right) \delta / \mathrm{ppm}$ : 9.87 $(\mathrm{s}, 1 \mathrm{H},-\mathrm{CHO}), 7.67\left(\mathrm{~d}, 1 \mathrm{H}, J=3.9 \mathrm{~Hz}, \mathrm{H}_{\mathrm{E} / \mathrm{F}}\right), 7.43\left(\mathrm{~d}, 2 \mathrm{H}, J=8.4 \mathrm{~Hz}, \mathrm{H}_{\mathrm{A} / \mathrm{B}}\right), 7.35(\mathrm{~d}, 2 \mathrm{H}$, $\left.J=8.4 \mathrm{~Hz}, \mathrm{H}_{\mathrm{A} / \mathrm{B}}\right), 7.19\left(\mathrm{~d}, 1 \mathrm{H}, J=16.1 \mathrm{~Hz}, \mathrm{H}_{\mathrm{C} / \mathrm{D}}\right), 7.16\left(\mathrm{~d}, 1 \mathrm{H}, J=3.9 \mathrm{~Hz}, \mathrm{H}_{\mathrm{E} / \mathrm{F}}\right), 7.09(\mathrm{~d}, 1 \mathrm{H}$, $\left.J=16.1 \mathrm{~Hz}, \mathrm{H}_{\mathrm{C} / \mathrm{D}}\right)$;

MS (ESI) $m / z$ (\%, fragment): 249/251 (100, $\left.\mathrm{M}+\mathrm{H}^{+}\right), 121$ (30); HRMS $(\mathrm{m} / z)$ for $\mathrm{C}_{13} \mathrm{H}_{9} \mathrm{ClOS}$ (obtained for the pure mixture of geometrical isomers): $[\mathrm{M}+\mathrm{H}]^{+}$calcd $=249.0061,[\mathrm{M}+\mathrm{H}]^{+}$measured $=249.0063$.<smiles>Cc1bcccc1-c1ccc(C=O)s1</smiles>

cis-10



trans-10 
cis-4-(2-(5-formylthiophen-2-yl)vinyl)benzonitrile (cis-10): obtained only in traces; $R_{f}$ (petroleum ether/DCM $(50 \%))=0,17$;

trans-4-(2-(5-formylthiophen-2-yl)vinyl)benzonitrile (trans-10): $472 \mathrm{mg}(29.4 \%) ; R_{f}$ (petroleum ether/DCM $(50 \%))=0.16 ;{ }^{1} \mathrm{H}$ NMR $\left(\mathrm{CDCl}_{3}, 600 \mathrm{MHz}\right) \delta / \mathrm{ppm}: 9.90(\mathrm{~s}, 1 \mathrm{H}$, - $\underline{\mathrm{HO}}$ ), $7.70\left(\mathrm{~d}, 1 \mathrm{H}, J=3.9 \mathrm{~Hz}, \mathrm{H}_{\mathrm{E} / \mathrm{F}}\right), 7.67\left(\mathrm{~d}, 2 \mathrm{H}, J=8.3 \mathrm{~Hz}, \mathrm{H}_{\mathrm{A} / \mathrm{B}}\right), 7.59(\mathrm{~d}, 2 \mathrm{H}, J=8.3 \mathrm{~Hz}$, $\left.\mathrm{H}_{\mathrm{A} / \mathrm{B}}\right), 7.32\left(\mathrm{~d}, 1 \mathrm{H}, J=16.1 \mathrm{~Hz}, \mathrm{H}_{\mathrm{C} / \mathrm{D}}\right), 7.23\left(\mathrm{~d}, 1 \mathrm{H}, J=3.9 \mathrm{~Hz}, \mathrm{H}_{\mathrm{E} / \mathrm{F}}\right), 7.12(\mathrm{~d}, 1 \mathrm{H}, J=16,1 \mathrm{~Hz}$, $\mathrm{H}_{\mathrm{C} / \mathrm{D})}$;

MS (ESI) $m / z\left(\%\right.$, fragment): $240\left(100, \mathrm{M}+\mathrm{H}^{+}\right)$; HRMS $(m / z)$ for $\mathrm{C}_{14} \mathrm{H}_{9} \mathrm{NOS}$ (obtained for the pure mixture of geometrical isomers): $[\mathrm{M}+\mathrm{H}]^{+}{ }_{\text {calcd }}=240.1331,[\mathrm{M}+\mathrm{H}]^{+}$measured $=240.1335$.<smiles>O=Cc1ccc(-c2ccc([N+](=O)[O-])cc2)s1</smiles>

cis-11

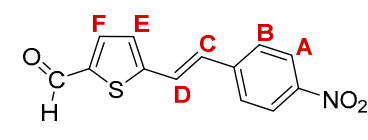

trans-11

cis-5-(4-nitrostyryl)thiophene-2-carbaldehyde (cis-11): $122 \mathrm{mg}$ (9.0\%); $R_{f}$ (petroleum ether/ diethyl ether $(50 \%))=0.25 ;{ }^{1} \mathrm{H}$ NMR $\left(\mathrm{CDCl}_{3}, 600 \mathrm{MHz}\right) \delta / \mathrm{ppm}$ : $9.82(\mathrm{~s}, 1 \mathrm{H},-\mathrm{C} \underline{\mathrm{HO}}), 8.23(\mathrm{~d}$, $\left.2 \mathrm{H}, J=8.7 \mathrm{~Hz}, \mathrm{H}_{\mathrm{A} / \mathrm{B}}\right), 7.60\left(\mathrm{~d}, 2 \mathrm{H}, J=8.7 \mathrm{~Hz}, \mathrm{H}_{\mathrm{A} / \mathrm{B}}\right), 7.57\left(\mathrm{~d}, 1 \mathrm{H}, J=3.9 \mathrm{~Hz}, \mathrm{H}_{\mathrm{E} / \mathrm{F}}\right), 7.03(\mathrm{~d}, 1 \mathrm{H}$, $\left.J=3.9 \mathrm{~Hz}, \mathrm{H}_{\mathrm{E} / \mathrm{F}}\right), 6.85\left(\mathrm{~d}, 1 \mathrm{H}, J=12.2 \mathrm{~Hz}, \mathrm{H}_{\mathrm{C} / \mathrm{D}}\right), 6.80\left(\mathrm{~d}, 1 \mathrm{H}, J=12.2 \mathrm{~Hz}, \mathrm{H}_{\mathrm{C} / \mathrm{D}}\right)$;

trans-5-(4-nitrostyryl)thiophene-2-carbaldehyde (trans-11) [33]: $244 \mathrm{mg}(18.0 \%) ; R_{f}$ (petroleter/diethyl ether $(50 \%))=0.20 ;{ }^{1} \mathrm{H} \mathrm{NMR}\left(\mathrm{CDCl}_{3}, 600 \mathrm{MHz}\right) \delta / \mathrm{ppm}: 9.91(\mathrm{~s}, 1 \mathrm{H},-\mathrm{C} \underline{\mathrm{HO}})$, $8.25\left(\mathrm{~d}, 2 \mathrm{H}, J=8.6 \mathrm{~Hz}, \mathrm{H}_{\mathrm{A} / \mathrm{B}}\right), 7.71\left(\mathrm{~d}, 1 \mathrm{H}, J=4.0 \mathrm{~Hz}, \mathrm{H}_{\mathrm{E} / \mathrm{F}}\right), 7.65\left(\mathrm{~d}, 2 \mathrm{H}, J=8.6 \mathrm{~Hz}, \mathrm{H}_{\mathrm{A} / \mathrm{B}}\right)$, $7.37\left(\mathrm{~d}, 1 \mathrm{H}, J=16.2 \mathrm{~Hz}, \mathrm{H}_{\mathrm{C} / \mathrm{D}}\right), 7.26\left(\mathrm{~d}, 1 \mathrm{H}, J=4.0 \mathrm{~Hz}, \mathrm{H}_{\mathrm{E} / \mathrm{F}}\right), 7.18(\mathrm{~d}, 1 \mathrm{H}, J=16.2 \mathrm{~Hz}$, $\mathrm{H}_{\mathrm{C} / \mathrm{D})}$;

MS (ESI) $m / z$ (\%, fragment): $260\left(100, \mathrm{M}+\mathrm{H}^{+}\right)$; $\mathrm{HRMS}(\mathrm{m} / \mathrm{z})$ for $\mathrm{C}_{13} \mathrm{H}_{9} \mathrm{NO}_{3} \mathrm{~S}$ (obtained for the pure mixture of geometrical isomers): $[\mathrm{M}+\mathrm{H}]^{+}$calcd $=260.0303,[\mathrm{M}+\mathrm{H}]^{+}$measured $=260.0306$.<smiles></smiles><smiles></smiles>

cis-5-(4-(dimethylamino)styryl)thiophene-2-carbaldehyde (cis-12): $247 \mathrm{mg}(19.1 \%) ; R_{f}$ $(\mathrm{DCM})=0.51 ;{ }^{1} \mathrm{H}$ NMR $\left(\mathrm{CDCl}_{3}, 600 \mathrm{MHz}\right) \delta / \mathrm{ppm}: 9.63(\mathrm{~s}, 1 \mathrm{H},-\mathrm{CHO}), 7.02(\mathrm{~d}, 1 \mathrm{H}$, mboxemphJ $\left.=3.4 \mathrm{~Hz}, \mathrm{H}_{\mathrm{E} / \mathrm{F}}\right), 6.70\left(\mathrm{~d}, 1 \mathrm{H}, J=3.4 \mathrm{~Hz}, \mathrm{H}_{\mathrm{E} / \mathrm{F}}\right), 6.68\left(\mathrm{~d}, 1 \mathrm{H}, J=11.8 \mathrm{~Hz}, \mathrm{H}_{\mathrm{C} / \mathrm{D}}\right)$, $6.66\left(\mathrm{~d}, 2 \mathrm{H}, J=8.7 \mathrm{~Hz}, \mathrm{H}_{\mathrm{A} / \mathrm{B}}\right), 6.55\left(\mathrm{~d}, 2 \mathrm{H}, J=8.7 \mathrm{~Hz}, \mathrm{H}_{\mathrm{A} / \mathrm{B}}\right), 6.49\left(\mathrm{~d}, 1 \mathrm{H}, J=11.8 \mathrm{~Hz}, \mathrm{H}_{\mathrm{C} / \mathrm{D}}\right)$, $2.98\left(\mathrm{~s}, 6 \mathrm{H},-\mathrm{N}\left(\mathrm{CH}_{3}\right)_{2}\right)$;

trans-5-(4-(dimethylamino)styryl)thiophene-2-carbaldehyde (trans-12) [34]: $227 \mathrm{mg}$ (27.5\%); $R_{f}(\mathrm{DCM})=0.44 ;{ }^{1} \mathrm{H} \mathrm{NMR}\left(\mathrm{CDCl}_{3}, 600 \mathrm{MHz}\right) \delta / \mathrm{ppm}: 9.80(\mathrm{~s}, 1 \mathrm{H},-\mathrm{C} \underline{\mathrm{HO}}), 7.56(\mathrm{~d}, 1 \mathrm{H}$, $\left.J=4.0 \mathrm{~Hz}, \mathrm{H}_{\mathrm{E} / \mathrm{F}}\right), 7.25\left(\mathrm{~d}, 2 \mathrm{H}, J=8.7 \mathrm{~Hz}, \mathrm{H}_{\mathrm{A} / \mathrm{B}}\right), 7.23\left(\mathrm{~d}, 2 \mathrm{H}, J=8.7 \mathrm{~Hz}, \mathrm{H}_{\mathrm{A} / \mathrm{B}}\right), 7.10(\mathrm{~d}, 1 \mathrm{H}$, $\left.J=16.5 \mathrm{~Hz}, \mathrm{H}_{\mathrm{C} / \mathrm{D}}\right), 7.09\left(\mathrm{~d}, 1 \mathrm{H}, J=4.0 \mathrm{~Hz}, \mathrm{H}_{\mathrm{E} / \mathrm{F}}\right), 7.00\left(\mathrm{~d}, 1 \mathrm{H}, J=16.5 \mathrm{~Hz}, \mathrm{H}_{\mathrm{C} / \mathrm{D}}\right), 2.99(\mathrm{~s}, 6 \mathrm{H}$, $\left.-\mathrm{N}\left(\underline{\mathrm{CH}_{3}}\right)_{2}\right)$;

MS (ESI) $m / z$ (\%, fragment): $258\left(100, \mathrm{M}+\mathrm{H}^{+}\right)$; HRMS $(\mathrm{m} / z)$ for $\mathrm{C}_{15} \mathrm{H}_{15} \mathrm{NOS}$ (obtained for the pure mixture of geometrical isomers): $[\mathrm{M}+\mathrm{H}]^{+}{ }_{\text {calcd }}=258.0874,[\mathrm{M}+\mathrm{H}]^{+}$measured $=258.0879$.

\subsection{Synthesis of Oximes 13-18}

The obtained mixture of cis- and trans-isomers of aldehydes, products of Vilsmeier formylations 7-12, were converted into corresponding oximes according to the literature [25]. Crystals of $\mathrm{NH}_{2} \mathrm{OH} \times \mathrm{HCl}$ were dissolved in a prepared mixture of $10 \mathrm{~mL}$ of $\mathrm{EtOH}$ and $3 \mathrm{~mL}$ of distilled water. After a homogeneous solution was obtained, the 
corresponding prepared heterostilbene aldehyde derivative 7-12 was added. The reaction mixture was stirred at room temperature for $24 \mathrm{~h}$. When the reaction was completed, the solvent was evaporated on a rotavapor under reduced pressure. The reaction mixture was purified by repeated column chromatography on silica gel using PE/DCM and $\mathrm{DCM} /$ methanol variable polarity eluents. The desired oxime derivatives $\mathbf{1 3 - 1 8}$ as pure geometrical isomers were thus isolated. The spectroscopic data and yields of pure isolated isomers of compounds 13-18 are given below.

5-(4-methylstyryl)thiophene-2-carbaldehyde oxime. Yield $465 \mathrm{mg}(87 \%)$; according to ${ }^{1} \mathrm{H}$ NMR spectroscopy the ratio of isomers in the mixture is cis,syn-: trans,syn-: trans,anti- = $1: 3: 15$;

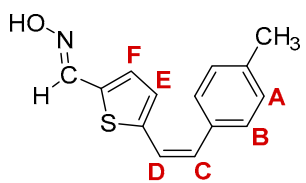

cis,syn-13<smiles>Cc1ccc(C=Cc2cc(F)c(C=NO)s2)cc1</smiles>

trans,syn-13



trans,anti-13

cis,syn-5-(4-methylstyryl)thiophene-2-carbaldehyde oxime (cis,syn-13): $5 \mathrm{mg} ; R_{f}$ (petroleum ether/diethyl ether $(30 \%))=0.33 ;{ }^{1} \mathrm{H} \mathrm{NMR}\left(\mathrm{CDCl}_{3}, 600 \mathrm{MHz}\right) \delta / \mathrm{ppm}: 8.14(\mathrm{~s}$, $1 \mathrm{H},-\mathrm{CH}=\mathrm{NOH}), 7.25\left(\mathrm{~d}, 2 \mathrm{H}, J=7.6 \mathrm{~Hz}, \mathrm{H}_{\mathrm{A} / \mathrm{B}}\right), 7.15\left(\mathrm{~d}, 2 \mathrm{H}, J=7.6 \mathrm{~Hz}, \mathrm{H}_{\mathrm{A} / \mathrm{B}}\right), 6.98(\mathrm{~d}, 1 \mathrm{H}$, $\left.J=3.6 \mathrm{~Hz}, \mathrm{H}_{\mathrm{E} / \mathrm{F}}\right), 6.89\left(\mathrm{~d}, 1 \mathrm{H}, J=3.6 \mathrm{~Hz}, \mathrm{H}_{\mathrm{E} / \mathrm{F}}\right), 6.61\left(\mathrm{~d}, 1 \mathrm{H}, J=12.3 \mathrm{~Hz}, \mathrm{H}_{\mathrm{C} / \mathrm{D}}\right), 6.58(\mathrm{~d}, 1 \mathrm{H}$, $\left.J=12.3 \mathrm{~Hz}, \mathrm{H}_{\mathrm{C} / \mathrm{D}}\right), 2.17\left(\mathrm{~s}, 3 \mathrm{H},-\mathrm{CH}_{3}\right)$;

trans,syn-5-(4-methylstyryl)thiophene-2-carbaldehyde oxime (trans,syn-13): $55 \mathrm{mg} ; R_{f}$ (petroleum ether/diethyl ether $(30 \%))=0.34 ;{ }^{1} \mathrm{H} \mathrm{NMR}\left(\mathrm{CDCl}_{3}, 600 \mathrm{MHz}\right) \delta / \mathrm{ppm}: 8.22(\mathrm{~s}$, $1 \mathrm{H},-\mathrm{CH}=\mathrm{NOH}), 7.47(\mathrm{bs}, 1 \mathrm{H},=\mathrm{N}-\mathrm{OH}), 7.36\left(\mathrm{~d}, 2 \mathrm{H}, J=7.9 \mathrm{~Hz}, \mathrm{H}_{\mathrm{A} / \mathrm{B}}\right), 7.16(\mathrm{~d}, 2 \mathrm{H}, J=7.9$ $\left.\mathrm{Hz}, \mathrm{H}_{\mathrm{A} / \mathrm{B}}\right), 7.12\left(\mathrm{~d}, 1 \mathrm{H}, J=15.9 \mathrm{~Hz}, \mathrm{H}_{\mathrm{C} / \mathrm{D}}\right), 7.08\left(\mathrm{~d}, 1 \mathrm{H}, J=3.5 \mathrm{~Hz}, \mathrm{H}_{\mathrm{E} / \mathrm{F}}\right), 6.96(\mathrm{~d}, 1 \mathrm{H}, J=3.5$ $\left.\mathrm{Hz}, \mathrm{H}_{\mathrm{E} / \mathrm{F}}\right), 6.94\left(\mathrm{~d}, 1 \mathrm{H}, J=15.9 \mathrm{~Hz}, \mathrm{H}_{\mathrm{C} / \mathrm{D}}\right), 2.36\left(\mathrm{~s}, 3 \mathrm{H},-\mathrm{CH}_{3}\right) ;{ }^{13} \mathrm{C} \mathrm{NMR}\left(\mathrm{CDCl}_{3}, 150 \mathrm{MHz}\right)$

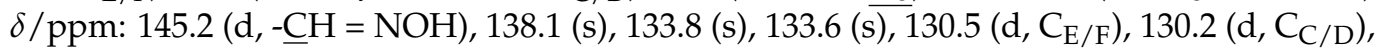
$129.5\left(2 \mathrm{~d}, \mathrm{C}_{\mathrm{A} / \mathrm{B}}\right), 126.5(\mathrm{~s}), 126.4\left(2 \mathrm{~d}, \mathrm{C}_{\mathrm{A} / \mathrm{B}}\right), 125.9\left(\mathrm{~d}, \mathrm{C}_{\mathrm{E} / \mathrm{F}}\right), 120.4\left(\mathrm{~d}, \mathrm{C}_{\mathrm{C} / \mathrm{D}}\right), 21.4\left(\mathrm{q},-\mathrm{CH}_{3}\right)$;

trans, anti-5-(4-methylstyryl)thiophene-2-carbaldehyde oxime (trans,anti-13): $67 \mathrm{mg} ; R_{f}$ (petroleum ether/diethyl ether $(30 \%))=0.20 ;{ }^{1} \mathrm{H}$ NMR $\left(\mathrm{CDCl}_{3}, 600 \mathrm{MHz}\right) \delta / \mathrm{ppm}: 7.64(\mathrm{~s}$, $1 \mathrm{H},-\underline{\mathrm{H}}=\mathrm{NOH}), 7.38\left(\mathrm{~d}, 2 \mathrm{H}, J=8.1 \mathrm{~Hz}, \mathrm{H}_{\mathrm{A} / \mathrm{B}}\right), 7.17\left(\mathrm{~d}, 1 \mathrm{H}, J=16.0 \mathrm{~Hz}, \mathrm{H}_{\mathrm{C} / \mathrm{D}}\right), 7.17(\mathrm{~d}, 2 \mathrm{H}$, $\left.J=8.1 \mathrm{~Hz}, \mathrm{H}_{\mathrm{A} / \mathrm{B}}\right), 7.16\left(\mathrm{~d}, 1 \mathrm{H}, J=3.5 \mathrm{~Hz}, \mathrm{H}_{\mathrm{E} / \mathrm{F}}\right), 7.04\left(\mathrm{~d}, 1 \mathrm{H}, J=16.0 \mathrm{~Hz}, \mathrm{H}_{\mathrm{C} / \mathrm{D}}\right), 7.03(\mathrm{~d}, 1 \mathrm{H}$, $\left.J=3.5 \mathrm{~Hz}, \mathrm{H}_{\mathrm{E} / \mathrm{F}}\right), 2.36\left(\mathrm{~s}, 3 \mathrm{H},-\mathrm{CH}_{3}\right) ;{ }^{13} \mathrm{C} \mathrm{NMR}\left(\mathrm{CDCl}_{3}, 150 \mathrm{MHz}\right) \delta / \mathrm{ppm}: 141.8(\mathrm{~d},-\underline{\mathrm{CH}}=$ $\mathrm{NOH}), 138.1$ (s), 133.8 (s), 132.6 (d), 130.2 (d, $\left.\mathrm{C}_{\mathrm{C} / \mathrm{D}}\right), 129.5$ (2d, $\left.\mathrm{C}_{\mathrm{A} / \mathrm{B}}\right), 128.9$ (s), 126.5 (2d, $\left.\mathrm{C}_{\mathrm{A} / \mathrm{B}}\right), 126.5(\mathrm{~s}), 124.8(\mathrm{~s}), 120.4\left(\mathrm{~d}, \mathrm{C}_{\mathrm{C} / \mathrm{D}}\right), 21.4\left(\mathrm{q},-\underline{\mathrm{CH}}_{3}\right)$;

MS (ESI) $m / z$ (\%, fragment): $244\left(100, \mathrm{M}+\mathrm{H}^{+}\right)$; $\mathrm{HRMS}(\mathrm{m} / z)$ for $\mathrm{C}_{14} \mathrm{H}_{13} \mathrm{NOS}$ (obtained for the pure mixture of geometrical isomers): $[\mathrm{M}+\mathrm{H}]^{+}$calcd $=244.0718,[\mathrm{M}+\mathrm{H}]^{+}$measured $=244.0716$.

5-(4-methoxystyryl)thiophene-2-carbaldehyde oxime. Yield $288 \mathrm{mg}$ (83\%); according to ${ }^{1} \mathrm{H}$ NMR spectroscopy the ratio of isomers in the mixture is cis,syn-:trans,syn-:trans,anti- = $1: 6: 20$;<smiles></smiles>

cis,syn-14<smiles>COc1ccc(/C=C/c2cc(F)c(/C=N/O)s2)cc1</smiles>

trans,syn-14



trans,anti-14

cis,syn-5-(4-methoxystyryl)thiophene-2-carbaldehyde oxime (cis,syn-14): $5 \mathrm{mg} ; R_{f}(\mathrm{DCM})$ $=0.38 ;{ }^{1} \mathrm{H}$ NMR $\left(\mathrm{CDCl}_{3}, 600 \mathrm{MHz}\right) \delta / \mathrm{ppm}: 8.15(\mathrm{~s}, 1 \mathrm{H},-\mathrm{CH}=\mathrm{NOH}), 7.29(\mathrm{~d}, 2 \mathrm{H}, \mathrm{J}=8.6 \mathrm{~Hz}$, $\left.\mathrm{H}_{\mathrm{A} / \mathrm{B}}\right), 7.10$ (bs, $\left.1 \mathrm{H},-\mathrm{CH}=\mathrm{N}-\mathrm{OH}\right), 6.98\left(\mathrm{~d}, 1 \mathrm{H}, J=3,3 \mathrm{~Hz}, \mathrm{H}_{\mathrm{E} / \mathrm{F}}\right), 6.90(\mathrm{~d}, 1 \mathrm{H}, J=3,3 \mathrm{~Hz}$, $\left.\mathrm{H}_{\mathrm{E} / \mathrm{F}}\right), 6.88\left(\mathrm{~d}, 2 \mathrm{H}, J=8.6 \mathrm{~Hz}, \mathrm{H}_{\mathrm{A} / \mathrm{B}}\right), 6.58\left(\mathrm{~d}, 1 \mathrm{H}, J=12.0 \mathrm{~Hz}, \mathrm{H}_{\mathrm{C} / \mathrm{D}}\right), 6.55(\mathrm{~d}, 1 \mathrm{H}, J=12.0 \mathrm{~Hz}$, 
$\left.\mathrm{H}_{\mathrm{C} / \mathrm{D}}\right), 3.83\left(\mathrm{~s}, 3 \mathrm{H},-\mathrm{OCH}_{3}\right) ;{ }^{13} \mathrm{C} \mathrm{NMR}\left(\mathrm{CDCl}_{3}, 150 \mathrm{MHz}\right) \delta / \mathrm{ppm}: 144.7(\mathrm{~d},-\mathrm{C} \mathrm{H}=\mathrm{NOH})$, $134.0(\mathrm{~s}), 132.7$ (s), $129.5(2 \mathrm{~d}), 128.8$ (d), 128.7 (s), 128.5 (s), 127.3 (2d), $124.9(\mathrm{~d}), 118.8$ (d), $113.7(\mathrm{~d}), 54.7(\mathrm{q})$;

trans,syn-5-(4-methoxystyryl)thiophene-2-carbaldehyde oxime (trans,syn-14): $68 \mathrm{mg} ; R_{f}$ $(\mathrm{DCM})=0.38 ;{ }^{1} \mathrm{H}$ NMR $\left(\mathrm{CDCl}_{3}, 600 \mathrm{MHz}\right) \delta / \mathrm{ppm}: 8.22(\mathrm{~s}, 1 \mathrm{H},-\mathrm{C} \underline{\mathrm{H}}=\mathrm{NOH}), 7.54(\mathrm{bs}, 1 \mathrm{H},=$ $\mathrm{N}-\mathrm{OH}), 7.41\left(\mathrm{~d}, 2 \mathrm{H}, J=8.6 \mathrm{~Hz}, \mathrm{H}_{\mathrm{A} / \mathrm{B}}\right), 7.07\left(\mathrm{~d}, 1 \mathrm{H}, J=3.8 \mathrm{~Hz}, \mathrm{H}_{\mathrm{E} / \mathrm{F}}\right), 7.04(\mathrm{~d}, 1 \mathrm{H}, J=16.1 \mathrm{~Hz}$, $\left.\mathrm{H}_{\mathrm{C} / \mathrm{D}}\right), 6.94\left(\mathrm{~d}, 1 \mathrm{H}, J=3.8 \mathrm{~Hz}, \mathrm{H}_{\mathrm{E} / \mathrm{F}}\right), 6.93\left(\mathrm{~d}, 1 \mathrm{H}, J=16.1 \mathrm{~Hz}, \mathrm{H}_{\mathrm{C} / \mathrm{D}}\right), 6.89(\mathrm{~d}, 2 \mathrm{H}, J=8.6 \mathrm{~Hz}$, $\left.\mathrm{H}_{\mathrm{A} / \mathrm{B}}\right), 3.83\left(\mathrm{~s}, 3 \mathrm{H},-\mathrm{OCH}_{3}\right) ;{ }^{13} \mathrm{C}$ NMR $\left(\mathrm{CDCl}_{3}, 150 \mathrm{MHz}\right) \delta / \mathrm{ppm}$ : $145.2(\mathrm{~d},-\mathrm{CH}=\mathrm{NOH})$,

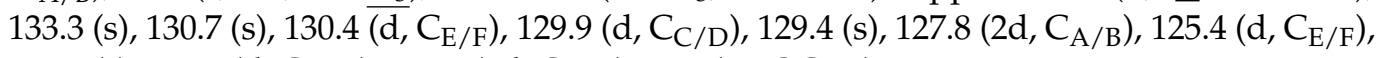
$122.0(\mathrm{~s}), 119.3\left(\mathrm{~d}, \mathrm{C}_{\mathrm{C} / \mathrm{D}}\right), 114.3\left(2 \mathrm{~d}, \mathrm{C}_{\mathrm{A} / \mathrm{B}}\right), 55.2\left(\mathrm{q},-\mathrm{OCH}_{3}\right)$;

trans,anti-5-(4-methoxystyryl)thiophene-2-carbaldehyde oxime (trans,anti-14): $93 \mathrm{mg} ; R_{f}$ $(\mathrm{DCM})=0.19 ;{ }^{1} \mathrm{H}$ NMR $\left(\mathrm{CDCl}_{3}, 600 \mathrm{MHz}\right) \delta / \mathrm{ppm}: 7.64(\mathrm{~s}, 1 \mathrm{H},-\mathrm{C} \underline{\mathrm{H}}=\mathrm{NOH}), 7.42(\mathrm{~d}, 2 \mathrm{H}$, $\left.J=8.6 \mathrm{~Hz}, \mathrm{H}_{\mathrm{A} / \mathrm{B}}\right), 7.26\left(\mathrm{~d}, 1 \mathrm{H}, J=4.0 \mathrm{~Hz}, \mathrm{H}_{\mathrm{E} / \mathrm{F}}\right), 7.09\left(\mathrm{~d}, 1 \mathrm{H}, J=16.2 \mathrm{~Hz}, \mathrm{H}_{\mathrm{C} / \mathrm{D}}\right), 7.02(\mathrm{~d}, 1 \mathrm{H}$, $\left.J=16.0 \mathrm{~Hz}, \mathrm{H}_{\mathrm{C} / \mathrm{D}}\right), 7.01\left(\mathrm{~d}, 1 \mathrm{H}, J=4.0 \mathrm{~Hz}, \mathrm{H}_{\mathrm{E} / \mathrm{F}}\right), 6.90\left(\mathrm{~d}, 2 \mathrm{H}, J=8.6 \mathrm{~Hz}, \mathrm{H}_{\mathrm{A} / \mathrm{B}}\right), 4.62(\mathrm{bs}$, $1 \mathrm{H},-\mathrm{CH}=\mathrm{NOH}), 3.83\left(\mathrm{~s}, 3 \mathrm{H},-\mathrm{OCH}_{3}\right) ;{ }^{13} \mathrm{C} \mathrm{NMR}\left(\mathrm{CDCl}_{3}, 150 \mathrm{MHz}\right) \delta / \mathrm{ppm}: 141.2(\mathrm{~d},-\underline{\mathrm{CH}}=$ $\mathrm{NOH}), 133.3(\mathrm{~s}), 132.6(\mathrm{~s}), 132.1\left(\mathrm{~d}, \overline{\mathrm{C}_{\mathrm{E}} / \mathrm{F}}\right), 129.4\left(\mathrm{~d}, \mathrm{C}_{\mathrm{C} / \mathrm{D}}\right), 128.9(\mathrm{~s}), 128.7(\mathrm{~s}), 127.4\left(2 \mathrm{~d}, \mathrm{C}_{\mathrm{A} / \mathrm{B}}\right)$, $124.0\left(\mathrm{~d}, \mathrm{C}_{\mathrm{E} / \mathrm{F}}\right), 118.8\left(\mathrm{~d}, \mathrm{C}_{\mathrm{C} / \mathrm{D}}\right), 113.8\left(2 \mathrm{~d}, \mathrm{C}_{\mathrm{A} / \mathrm{B}}\right), 54.9\left(\mathrm{q},-\mathrm{OCH}_{3}\right)$;

MS (ESI) $m / z$ (\%, fragment): $260\left(100, \mathrm{M}+\mathrm{H}^{+}\right)$; HRMS $(\mathrm{m} / z)$ for $\mathrm{C}_{14} \mathrm{H}_{13} \mathrm{NO}_{2} \mathrm{~S}$ (obtained for the pure mixture of geometrical isomers): $[\mathrm{M}+\mathrm{H}]^{+}$calcd $=260.0667,[\mathrm{M}+\mathrm{H}]^{+}{ }_{\text {measured }}=$ 260.0666.

5-(4-chlorostyryl)thiophene-2-carbaldehyde oxime. Yield $363 \mathrm{mg}(77 \%)$; according to ${ }^{1} \mathrm{H}$ NMR spectroscopy the ratio of isomers in the mixture is cis,syn-:trans,syn-:trans,anti- = 1:5:13;<smiles>O/N=C/c1cc(F)c(/C=C\c2bcc(Cl)cc2)s1</smiles>

cis,syn-15<smiles>O/N=C/c1ccc(/C=C/c2bc(Cl)ccc2F)s1</smiles>

trans,syn-15



trans, anti-15

cis,syn-5-(4-chlorostyryl)thiophene-2-carbaldehyde oxime (cis,syn-15): $9 \mathrm{mg} ; R_{f}$ $(\mathrm{DCM} /$ methanol $(1 \%))=0.58 ;{ }^{1} \mathrm{H} \mathrm{NMR}\left(\mathrm{CDCl}_{3}, 600 \mathrm{MHz}\right) \delta / \mathrm{ppm}$ (data from the mixture with trans,syn-15): $8.15(\mathrm{~s}, 1 \mathrm{H},-\mathrm{CH}=\mathrm{NOH}), 7.32\left(\mathrm{~d}, 2 \mathrm{H}, J=8.5 \mathrm{~Hz}, \mathrm{H}_{\mathrm{A} / \mathrm{B}}\right), 7.29(\mathrm{~d}, 2 \mathrm{H}$, $\left.J=8.5 \mathrm{~Hz}, \mathrm{H}_{\mathrm{A} / \mathrm{B}}\right), 7.14\left(\mathrm{~d}, 1 \mathrm{H}, J=3.4 \mathrm{~Hz}, \mathrm{H}_{\mathrm{E} / \mathrm{F}}\right), 6.89\left(\mathrm{~d}, 1 \mathrm{H}, J=3.4 \mathrm{~Hz}, \mathrm{H}_{\mathrm{E} / \mathrm{F}}\right), 6.64(\mathrm{~d}, 1 \mathrm{H}$, $\left.J=12.1 \mathrm{~Hz}, \mathrm{H}_{\mathrm{C} / \mathrm{D}}\right), 6.56\left(\mathrm{~d}, 1 \mathrm{H}, J=12.1 \mathrm{~Hz}, \mathrm{H}_{\mathrm{C} / \mathrm{D}}\right)$;

trans,syn-5-(4-chlorostyryl)thiophene-2-carbaldehyde oxime (trans,syn-15): $56 \mathrm{mg}$; $R_{f}$ $(\mathrm{DCM} /$ methanol $(1 \%))=0.50 ;{ }^{1} \mathrm{H}$ NMR $\left(\mathrm{CDCl}_{3}, 600 \mathrm{MHz}\right) \delta / \mathrm{ppm}: 8.22(\mathrm{~s}, 1 \mathrm{H},-\mathrm{C} \underline{\mathrm{H}}=$ $\mathrm{NOH}), 7.39\left(\mathrm{~d}, 2 \mathrm{H}, J=8.5 \mathrm{~Hz}, \mathrm{H}_{\mathrm{A} / \mathrm{B}}\right), 7.31\left(\mathrm{~d}, 2 \mathrm{H}, J=8.5 \mathrm{~Hz}, \mathrm{H}_{\mathrm{A} / \mathrm{B}}\right), 7.14(\mathrm{bs}, 1 \mathrm{H},=\mathrm{N}-\mathrm{O} \underline{H})$, $7.14\left(\mathrm{~d}, 1 \mathrm{H}, J=16.2 \mathrm{~Hz}, \mathrm{H}_{\mathrm{C} / \mathrm{D}}\right), 7.09\left(\mathrm{~d}, 1 \mathrm{H}, J=3.7 \mathrm{~Hz}, \mathrm{H}_{\mathrm{E} / \mathrm{F}}\right), 6.99\left(\mathrm{~d}, 1 \mathrm{H}, J=3.7 \mathrm{~Hz}, \mathrm{H}_{\mathrm{E} / \mathrm{F}}\right)$, $6.91\left(\mathrm{~d}, 1 \mathrm{H}, J=16.2 \mathrm{~Hz}, \mathrm{H}_{\mathrm{C} / \mathrm{D}}\right) ;{ }^{13} \mathrm{C} \mathrm{NMR}\left(\mathrm{CDCl}_{3}, 150 \mathrm{MHz}\right) \delta / \mathrm{ppm}: 145.2(\mathrm{~d},-\underline{\mathrm{CH}}=\mathrm{NOH})$, $144.4(\mathrm{~s}), 135.1(\mathrm{~s}), 134.2(\mathrm{~s}), 133.6(\mathrm{~s}), 130.4\left(\mathrm{~d}, \mathrm{C}_{\mathrm{E} / \mathrm{F}}\right), 129.0\left(2 \mathrm{~d}, \mathrm{C}_{\mathrm{A} / \mathrm{B}}\right), 128.7\left(\mathrm{~d}, \mathrm{C}_{\mathrm{C} / \mathrm{D}}\right), 127.6$ $\left(2 \mathrm{~d}, \mathrm{C}_{\mathrm{A} / \mathrm{B}}\right), 126.5\left(\mathrm{~d}, \mathrm{C}_{\mathrm{E} / \mathrm{F}}\right), 121.9\left(\mathrm{~d}, \mathrm{C}_{\mathrm{C} / \mathrm{D}}\right)$;

trans,anti-5-(4-chlorostyryl)thiophene-2-carbaldehyde oxime (trans,anti-15): $94 \mathrm{mg} ; R_{f}$ $(\mathrm{DCM} /$ methanol $(1 \%))=0.20 ;{ }^{1} \mathrm{H} \mathrm{NMR}\left(\mathrm{CDCl}_{3}, 600 \mathrm{MHz}\right) \delta / \mathrm{ppm}: 7.66(\mathrm{~s}, 1 \mathrm{H},-\mathrm{C} \underline{\mathrm{H}}=$ $\mathrm{NOH}), 7.40\left(\mathrm{~d}, 2 \mathrm{H}, J=8.5 \mathrm{~Hz}, \mathrm{H}_{\mathrm{A} / \mathrm{B}}\right), 7.32\left(\mathrm{~d}, 2 \mathrm{H}, J=8.5 \mathrm{~Hz}, \mathrm{H}_{\mathrm{A} / \mathrm{B}}\right), 7.27(\mathrm{~d}, 1 \mathrm{H}, J=4.0 \mathrm{~Hz}$, $\left.\mathrm{H}_{\mathrm{E} / \mathrm{F}}\right), 7.19\left(\mathrm{~d}, 1 \mathrm{H}, J=16.1 \mathrm{~Hz}, \mathrm{H}_{\mathrm{C} / \mathrm{D}}\right), 7.06\left(\mathrm{~d}, 1 \mathrm{H}, J=4.0 \mathrm{~Hz}, \mathrm{H}_{\mathrm{E} / \mathrm{F}}\right), 7.00(\mathrm{~d}, 1 \mathrm{H}, J=16.1 \mathrm{~Hz}$, $\left.\mathrm{H}_{\mathrm{C} / \mathrm{D}}\right) ;{ }^{13} \mathrm{C} \mathrm{NMR}\left(\mathrm{CDCl}_{3}, 150 \mathrm{MHz}\right) \delta / \mathrm{ppm}: 141.6(\mathrm{~d},-\underline{\mathrm{CH}}=\mathrm{NOH}), 135.1(\mathrm{~s}), 133.7(\mathrm{~s})$, $132.5\left(\mathrm{~d}, \mathrm{C}_{\mathrm{E} / \mathrm{F}}\right), 130.1(\mathrm{~s}), 129.0\left(2 \mathrm{~d}, \mathrm{C}_{\mathrm{A} / \mathrm{B}}\right), 128.8(\mathrm{~s}), 128.7\left(\mathrm{~d}, \mathrm{C}_{\mathrm{C} / \mathrm{D}}\right), 127.7\left(2 \mathrm{~d}, \mathrm{C}_{\mathrm{A} / \mathrm{B}}\right), 125.5$ $\left(\mathrm{d}, \mathrm{C}_{\mathrm{E} / \mathrm{F}}\right), 122.0\left(\mathrm{~d}, \mathrm{C}_{\mathrm{C} / \mathrm{D}}\right)$; 
MS (ESI) $m / z$ (\%, fragment): 264/266 (100, M+H $\left.{ }^{+}\right), 121$ (35); HRMS $(m / z)$ for $\mathrm{C}_{13} \mathrm{H}_{10} \mathrm{ClNOS}$ (obtained for the pure mixture of geometrical isomers): $[\mathrm{M}+\mathrm{H}]^{+}$calcd $=264.0172,[\mathrm{M}+\mathrm{H}]^{+}$measured $=264.0171$.

4-(2-(5-((hydroxyimino)methyl)thiophen-2-yl)vinyl)benzonitrile. Yield $366 \mathrm{mg}$ (73\%); according to ${ }^{1} \mathrm{H}$ NMR spectroscopy the ratio of isomers in the mixture is cis,syn-:trans,syn:trans, anti- = 1:30:10;<smiles>N#Cc1ccc(C=[C]c2cc(F)c(/C=N/O)s2)c(F)c1</smiles>

cis,syn-16

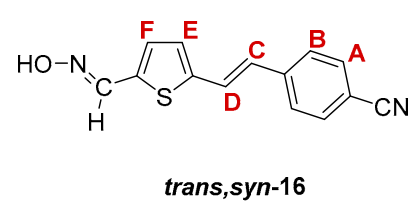

trans,syn-16

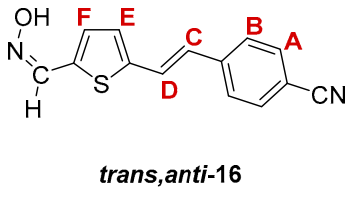

trans, anti-16

cis,syn-4-(2-(5-((hydroxyimino)methyl)thiophen-2-yl)vinyl)benzonitrile (cis,syn-16): $3 \mathrm{mg}$; $R_{f}(\mathrm{DCM} /$ methanol $(2.5 \%))=0.55 ;{ }^{1} \mathrm{H} \mathrm{NMR}\left(\mathrm{CDCl}_{3}, 600 \mathrm{MHz}\right) \delta / \mathrm{ppm}$ (data from the mixture with trans,syn-16): $8.14(\mathrm{~s}, 1 \mathrm{H},-\mathrm{CH}=\mathrm{NOH}), 7.64\left(\mathrm{~d}, 2 \mathrm{H}, J=8.1 \mathrm{~Hz}, \mathrm{H}_{\mathrm{A} / \mathrm{B}}\right), 7.47(\mathrm{~d}$, $\left.2 \mathrm{H}, J=8.1 \mathrm{~Hz}, \mathrm{H}_{\mathrm{A} / \mathrm{B}}\right), 7.35(\mathrm{~s}, 1 \mathrm{H},-\mathrm{CH}=\mathrm{NOH}), 6.99\left(\mathrm{~d}, 1 \mathrm{H}, J=3.7 \mathrm{~Hz}, \mathrm{H}_{\mathrm{E} / \mathrm{F}}\right), 6.89(\mathrm{~d}, 1 \mathrm{H}$, $\left.J=3.7 \mathrm{~Hz}, \mathrm{H}_{\mathrm{E} / \mathrm{F}}\right), 6.73\left(\mathrm{~d}, 1 \mathrm{H}, J=12.1 \mathrm{~Hz}, \mathrm{H}_{\mathrm{C} / \mathrm{D}}\right), 6.58\left(\mathrm{~d}, 1 \mathrm{H}, J=12.1 \mathrm{~Hz}, \mathrm{H}_{\mathrm{C} / \mathrm{D}}\right)$;

trans,syn-4-(2-(5-((hydroxyimino)methyl)thiophen-2-yl)vinyl)benzonitrile (trans,syn-16): $83 \mathrm{mg} ; R_{f}(\mathrm{DCM} /$ methanol $(2.5 \%))=0.55 ;{ }^{1} \mathrm{H} \mathrm{NMR}\left(\mathrm{CDCl}_{3}, 600 \mathrm{MHz}\right) \delta / \mathrm{ppm}: 8.23$ (s, $1 \mathrm{H},-\mathrm{CH}=\mathrm{NOH}), 7.62\left(\mathrm{~d}, 2 \mathrm{H}, J=8.4 \mathrm{~Hz}, \mathrm{H}_{\mathrm{A} / \mathrm{B}}\right), 7.53\left(\mathrm{~d}, 2 \mathrm{H}, J=8.4 \mathrm{~Hz}, \mathrm{H}_{\mathrm{A} / \mathrm{B}}\right), 7.43(\mathrm{~s}, 1 \mathrm{H},-\mathrm{CH}$ $=\mathrm{NO} \underline{\mathrm{H}}), 7.27\left(\mathrm{~d}, 1 \mathrm{H}, J=16.1 \mathrm{~Hz}, \mathrm{H}_{\mathrm{C} / \mathrm{D}}\right), 7.11\left(\mathrm{~d}, 1 \mathrm{H}, J=3.6 \mathrm{~Hz}, \mathrm{H}_{\mathrm{E} / \mathrm{F}}\right), 7.06(\mathrm{~d}, 1 \mathrm{H}, J=3.6 \mathrm{~Hz}$, $\left.\mathrm{H}_{\mathrm{E} / \mathrm{F}}\right), 6.93\left(\mathrm{~d}, 1 \mathrm{H}, J=16.1 \mathrm{~Hz}, \mathrm{H}_{\mathrm{C} / \mathrm{D}}\right) ;{ }^{13} \mathrm{C} \mathrm{NMR}\left(\mathrm{CDCl}_{3}, 150 \mathrm{MHz}\right) \delta / \mathrm{ppm}: 144.9(\mathrm{~d},-\underline{\mathrm{CH}}=$ $\mathrm{NOH}), 143.5$ (s), 141.1 (s), $135.4(\mathrm{~s}), 132.6\left(2 \mathrm{~d}, \mathrm{C}_{\mathrm{A} / \mathrm{B}}\right), 132.5(\mathrm{~s}), 130.4\left(\mathrm{~d}, \mathrm{C}_{\mathrm{E} / \mathrm{F}}\right), 127.9\left(\mathrm{~d}, \mathrm{C}_{\mathrm{C} / \mathrm{D}}\right)$, $127.8\left(\mathrm{~d}, \mathrm{C}_{\mathrm{E} / \mathrm{F}}\right), 126.8\left(2 \mathrm{~d}, \mathrm{C}_{\mathrm{A} / \mathrm{B}}\right), 124.8\left(\mathrm{~d}, \mathrm{C}_{\mathrm{C} / \mathrm{D}}\right), 118.9(\mathrm{~s},-\underline{\mathrm{CN}})$;

trans,anti-4-(2-(5-((hydroxyimino)methyl)thiophen-2-yl)vinyl)benzonitrile (trans,anti-16): $28 \mathrm{mg} ; R_{f}(\mathrm{DCM} / \mathrm{methanol}(2.5 \%))=0.27 ;{ }^{1} \mathrm{H} \mathrm{NMR}\left(\mathrm{CDCl}_{3}, 600 \mathrm{MHz}\right) \delta / \mathrm{ppm}$ : 7.68 (s, $1 \mathrm{H},-\mathrm{CH}=\mathrm{NOH}), 7.63\left(\mathrm{~d}, 2 \mathrm{H}, J=8.2 \mathrm{~Hz}, \mathrm{H}_{\mathrm{A} / \mathrm{B}}\right), 7.55\left(\mathrm{~d}, 2 \mathrm{H}, J=8.2 \mathrm{~Hz}, \mathrm{H}_{\mathrm{A} / \mathrm{B}}\right), 7.33(\mathrm{~d}, 1 \mathrm{H}$, $\left.J=16.2 \mathrm{~Hz}, \mathrm{H}_{\mathrm{C} / \mathrm{D}}\right), 7.29\left(\mathrm{~d}, 1 \mathrm{H}, J=4.0 \mathrm{~Hz}, \mathrm{H}_{\mathrm{E} / \mathrm{F}}\right), 7.13\left(\mathrm{~d}, 1 \mathrm{H}, J=4.0 \mathrm{~Hz}, \mathrm{H}_{\mathrm{E} / \mathrm{F}}\right), 7.03(\mathrm{~d}, 1 \mathrm{H}$, $\left.J=16.2 \mathrm{~Hz}, \mathrm{H}_{\mathrm{C} / \mathrm{D}}\right) ;{ }^{13} \mathrm{C} \mathrm{NMR}\left(\mathrm{CDCl}_{3}, 150 \mathrm{MHz}\right) \delta / \mathrm{ppm}: 141.4(\mathrm{~d},-\underline{\mathrm{CH}}=\mathrm{NOH}), 141.1(\mathrm{~s})$, $132.7(\mathrm{~s}), 132.6\left(2 \mathrm{~d}, \mathrm{C}_{\mathrm{A} / \mathrm{B}}\right), 132.5\left(\mathrm{~d}, \mathrm{C}_{\mathrm{E} / \mathrm{F}}\right), 129.6(\mathrm{~s}), 127.8\left(\mathrm{~d}, \mathrm{C}_{\mathrm{C} / \mathrm{D}}\right), 127.2(\mathrm{~s}), 126.8\left(2 \mathrm{~d}, \mathrm{C}_{\mathrm{A} / \mathrm{B}}\right)$, $126.7\left(\mathrm{~d}, \mathrm{C}_{\mathrm{E} / \mathrm{F}}\right), 124.9\left(\mathrm{~d}, \mathrm{C}_{\mathrm{C} / \mathrm{D}}\right), 118.9(\mathrm{~s},-\underline{\mathrm{CN}})$;

MS (ESI) $m / z$ (\%, fragment): $255\left(100, \mathrm{M}+\mathrm{H}^{+}\right)$; HRMS $(m / z)$ for $\mathrm{C}_{14} \mathrm{H}_{10} \mathrm{~N}_{2} \mathrm{OS}$ (obtained for the pure mixture of geometrical isomers): $[\mathrm{M}+\mathrm{H}]^{+}{ }_{\text {calcd }}=255.0514,[\mathrm{M}+\mathrm{H}]^{+}{ }_{\text {measured }}=$ 255.0512 .

5-(4-nitrostyryl)thiophene-2-carbaldehyde oxime. Yield $220 \mathrm{mg}$ (57\%); according to ${ }^{1} \mathrm{H}$ NMR spectroscopy the ratio of isomers in the mixture is trans,syn-:cis,anti-:trans, anti- = 8:1:5;

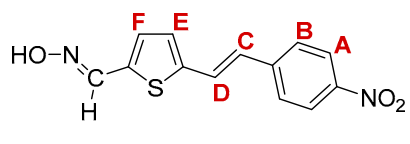

trans,syn-17

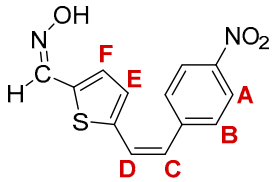

cis,anti-17

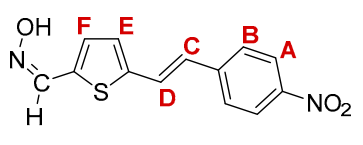

trans, anti-17

trans,syn-5-(4-nitrostyryl)thiophene-2-carbaldehyde oxime (trans,syn-17): $37 \mathrm{mg}$; $R_{f}(\mathrm{DCM} /$ methanol $(1 \%))=0.27 ;{ }^{1} \mathrm{H}$ NMR $\left(\mathrm{CDCl}_{3}, 600 \mathrm{MHz}\right) \delta / \mathrm{ppm}: 8.24(\mathrm{~s}, 1 \mathrm{H},-\mathrm{C} \underline{\mathrm{H}}=$ $\mathrm{NOH}), 8.21\left(\mathrm{~d}, 2 \mathrm{H}, J=8.8 \mathrm{~Hz}, \mathrm{H}_{\mathrm{A} / \mathrm{B}}\right), 7.59\left(\mathrm{~d}, 2 \mathrm{H}, J=8.8 \mathrm{~Hz}, \mathrm{H}_{\mathrm{A} / \mathrm{B}}\right), 7.33(\mathrm{~d}, 1 \mathrm{H}, J=16.2 \mathrm{~Hz}$, $\left.\mathrm{H}_{\mathrm{C} / \mathrm{D}}\right), 7.32(\mathrm{bs}, 1 \mathrm{H},-\mathrm{CH}=\mathrm{NO} \underline{\mathrm{H}}), 7.13\left(\mathrm{~d}, 1 \mathrm{H}, J=3.7 \mathrm{~Hz}, \mathrm{H}_{\mathrm{E} / \mathrm{F}}\right), 7.10(\mathrm{~d}, 1 \mathrm{H}, J=3.7 \mathrm{~Hz}$, $\left.\mathrm{H}_{\mathrm{E} / \mathrm{F}}\right), 6.98\left(\mathrm{~d}, 1 \mathrm{H}, J=16.2 \mathrm{~Hz}, \mathrm{H}_{\mathrm{C} / \mathrm{D}}\right) ;{ }^{13} \mathrm{C} \mathrm{NMR}\left(\mathrm{CDCl}_{3}, 150 \mathrm{MHz}\right) \delta / \mathrm{ppm}: 143.4$ (s), 141.5 (s), 133.7 (s), 132.5 (d), 128.2 (d), 127.3 (d), 126.9 (2d), 126.8 (s), 125.8 (d), 124.2 (2d), (1 signal is missing); 
cis,anti-5-(4-nitrostyryl)thiophene-2-carbaldehyde oxime (cis,anti-17): $6 \mathrm{mg} ; R_{f}(\mathrm{DCM} /$ methanol $(1 \%))=0,45 ;{ }^{1} \mathrm{H} \mathrm{NMR}\left(\mathrm{CDCl}_{3}, 600 \mathrm{MHz}\right) \delta / \mathrm{ppm}: 8.20\left(\mathrm{~d}, 2 \mathrm{H}, J=8.5 \mathrm{~Hz}, \mathrm{H}_{\mathrm{A} / \mathrm{B}}\right)$, $7.58(\mathrm{~s}, 1 \mathrm{H},-\mathrm{CH}=\mathrm{NOH}), 7.54\left(\mathrm{~d}, 2 \mathrm{H}, J=8.5 \mathrm{~Hz}, \mathrm{H}_{\mathrm{A} / \mathrm{B}}\right), 7.19\left(\mathrm{~d}, 1 \mathrm{H}, J=4.0 \mathrm{~Hz}, \mathrm{H}_{\mathrm{E} / \mathrm{F}}\right), 6.94(\mathrm{~d}$, $\left.1 \mathrm{H}, J=4.0 \mathrm{~Hz}, \mathrm{H}_{\mathrm{E} / \mathrm{F}}\right), 6.81\left(\mathrm{~d}, 1 \mathrm{H}, J=12.1 \mathrm{~Hz}, \mathrm{H}_{\mathrm{C} / \mathrm{D}}\right), 6.65\left(\mathrm{~d}, 1 \mathrm{H}, J=12.1 \mathrm{~Hz}, \mathrm{H}_{\mathrm{C} / \mathrm{D}}\right)$;

trans,anti-5-(4-nitrostyryl)thiophene-2-carbaldehyde oxime (trans,anti-17): $16 \mathrm{mg} ; R_{f}$ $(\mathrm{DCM} /$ methanol $(1 \%))=0.43 ;{ }^{1} \mathrm{H} \mathrm{NMR}\left(\mathrm{CDCl}_{3}, 600 \mathrm{MHz}\right) \delta / \mathrm{ppm}: 8.22(\mathrm{~d}, 2 \mathrm{H}, J=8.6 \mathrm{~Hz}$, $\left.\mathrm{H}_{\mathrm{A} / \mathrm{B}}\right), 7.68(\mathrm{~s}, 1 \mathrm{H},-\mathrm{CH}=\mathrm{NOH}), 7.60\left(\mathrm{~d}, 2 \mathrm{H}, J=8.6 \mathrm{~Hz}, \mathrm{H}_{\mathrm{A} / \mathrm{B}}\right), 7.38(\mathrm{~d}, 1 \mathrm{H}, J=16.2 \mathrm{~Hz}$, $\left.\mathrm{H}_{\mathrm{C} / \mathrm{D}}\right), 7.30\left(\mathrm{~d}, 1 \mathrm{H}, J=3.9 \mathrm{~Hz}, \mathrm{H}_{\mathrm{E} / \mathrm{F}}\right), 7.16\left(\mathrm{~d}, 1 \mathrm{H}, J=3.9 \mathrm{~Hz}, \mathrm{H}_{\mathrm{E} / \mathrm{F}}\right), 7.08(\mathrm{~d}, 1 \mathrm{H}, J=16.2 \mathrm{~Hz}$, $\mathrm{H}_{\mathrm{C} / \mathrm{D}}$ ); MS (ESI) $\mathrm{m} / z$ (\%, fragment): $275\left(100, \mathrm{M}+\mathrm{H}^{+}\right)$; HRMS $(\mathrm{m} / \mathrm{z})$ for $\mathrm{C}_{13} \mathrm{H}_{10} \mathrm{~N}_{2} \mathrm{O}_{3} \mathrm{~S}$ (obtained for the pure mixture of geometrical isomers): $[\mathrm{M}+\mathrm{H}]^{+}{ }_{\text {calcd }}=275.0412,[\mathrm{M}+\mathrm{H}]^{+}{ }_{\text {measured }}$ $=275.0413$.

5-(4-dimethylaminostyryl)thiophene-2-carbaldehyde oxime. Yield $436 \mathrm{mg}(88 \%)$; according to ${ }^{1} \mathrm{H}$ NMR spectroscopy the ratio of isomers in the mixture is cis,syn-:trans,syn:trans, anti- = 1:1:1;
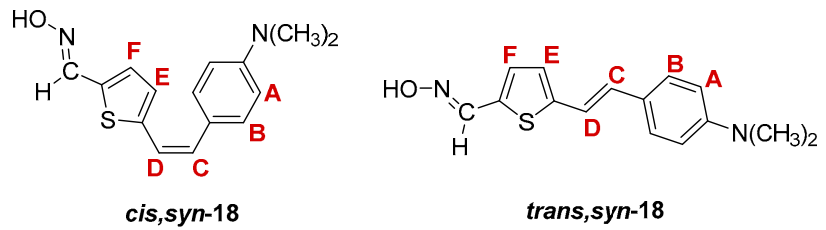

cys,syn-5-(4-dimethylaminostyryl)thiophene-2-carbaldehyde oxime (cys,syn-18): $75 \mathrm{mg} ; R_{f}(\mathrm{DCM} / \mathrm{methanol}(1 \%))=0.21 ;{ }^{1} \mathrm{H} \mathrm{NMR}\left(\mathrm{CDCl}_{3}, 600 \mathrm{MHz}\right) \delta / \mathrm{ppm}: 8.42(\mathrm{~s}$, $1 \mathrm{H},-\mathrm{C} \underline{\mathrm{H}}=\mathrm{NOH}), 7.34\left(\mathrm{~d}, 2 \mathrm{H}, J=8.8 \mathrm{~Hz}, \mathrm{H}_{\mathrm{A} / \mathrm{B}}\right), 7.31$ (broad s, $\left.1 \mathrm{H},-\mathrm{CH}=\mathrm{NO} \underline{\mathrm{H}}\right), 7.10(\mathrm{~d}$, $\left.1 \mathrm{H}, J=3.9 \mathrm{~Hz}, \mathrm{H}_{\mathrm{E} / \mathrm{F}}\right), 6.99\left(\mathrm{~d}, 2 \mathrm{H}, J=8.8 \mathrm{~Hz}, \mathrm{H}_{\mathrm{A} / \mathrm{B}}\right), 6.90\left(\mathrm{dd}, 1 \mathrm{H}, J=3.9 ; 1.9 \mathrm{~Hz}, \mathrm{H}_{\mathrm{E} / \mathrm{F}}\right), 6.65$ $\left(\mathrm{d}, 1 \mathrm{H}, J=11.9 \mathrm{~Hz}, \mathrm{H}_{\mathrm{C} / \mathrm{D}}\right), 6.48\left(\mathrm{~d}, 1 \mathrm{H}, J=11.9 \mathrm{~Hz}, \mathrm{H}_{\mathrm{C} / \mathrm{D}}\right), 2.77(\mathrm{~s}, 6 \mathrm{H}) ;{ }^{13} \mathrm{C} \mathrm{NMR}\left(\mathrm{CDCl}_{3}\right.$, $150 \mathrm{MHz}$ ) $\delta /$ ppm: 149.7 (s), 148.7 (d), 132.9 (s), 130.4 (d), 127.6 (2d), 127.6 (2d), 125.9 (d), $122.4(\mathrm{~d}), 117.8(\mathrm{~d}), 116.9(\mathrm{~s}), 108.8(\mathrm{~s}), 44.6(2 \mathrm{q})$;

trans,syn-5-(4-dimethylaminostyryl)thiophene-2-carbaldehyde oxime (trans,syn-18) (it was not possible to isolate this isomer without the traces of cis,syn-18): $R_{f}(\mathrm{DCM} / \mathrm{methanol}$ $(1 \%))=0.22 ;{ }^{1} \mathrm{H} \mathrm{NMR}\left(\mathrm{CDCl}_{3}, 600 \mathrm{MHz}\right) \delta / \mathrm{ppm}: 8.43(\mathrm{~s}, 1 \mathrm{H},-\mathrm{CH}=\mathrm{NOH}), 7.79$ (broad s, $1 \mathrm{H},-\mathrm{CH}=\mathrm{NO} \underline{\mathrm{H}}), 7.42\left(\mathrm{dd}, 1 \mathrm{H}, J=8.5 ; 2.0 \mathrm{~Hz}, \mathrm{H}_{\mathrm{A} / \mathrm{B}}\right), 7.17\left(\mathrm{~d}, 1 \mathrm{H}, J=4.1 \mathrm{~Hz}, \mathrm{H}_{\mathrm{E} / \mathrm{F}}\right), 7.15(\mathrm{~d}$, $\left.1 \mathrm{H}, J=15.6 \mathrm{~Hz}, \mathrm{H}_{\mathrm{C} / \mathrm{D}}\right), 7.07-6.97(\mathrm{~m}, 4 \mathrm{H}), 6.88\left(\mathrm{~d}, 1 \mathrm{H}, J=15.6 \mathrm{~Hz}, \mathrm{H}_{\mathrm{C} / \mathrm{D}}\right), 2.78(\mathrm{~s}, 6 \mathrm{H})$;

MS (ESI) $m / z$ (\%, fragment): $273\left(100, \mathrm{M}+\mathrm{H}^{+}\right)$; HRMS $(\mathrm{m} / z)$ for $\mathrm{C}_{15} \mathrm{H}_{16} \mathrm{~N}_{2} \mathrm{OS}$ (obtained for the pure mixture of geometrical isomers): $[\mathrm{M}+\mathrm{H}]^{+}$calcd $=273.0553,[\mathrm{M}+\mathrm{H}]^{+}{ }_{\text {measured }}=$ 273.0557.

\subsection{Chemicals for Enzyme Assays}

Recombinant human AChE was kindly donated by Dr Zoran Radić (Skaggs School of Pharmacy and Pharmaceutical Sciences, University of California at San Diego, La Jolla, CA, USA), while purified BChE isolated from human plasma was prepared and generously gifted by Dr Florain Nachon (Institut de Recherche Biomédicale des Armées, Bretignysur-Orge, France). Enzymes were diluted in $1 \%$ of BSA buffer and stored at $4{ }^{\circ} \mathrm{C}$ as work solutions. OP compounds sarin, cyclosarin, $\mathrm{VX}$, and tabun were purchased from the NC Laboratory (Spiez, Switzerland). OP stock solutions $(5000 \mu \mathrm{g} / \mathrm{mL})$ were made in isopropyl alcohol, and further dilutions were made in water just before use. The newly synthesized oximes were prepared in a DMSO solvent at a concentration of $100 \mathrm{mM}$ and stored at $4{ }^{\circ} \mathrm{C}$. Other reagents and solvents used in cholinesterase activity measurement were purchased from Sigma-Aldrich (St. Louis, MO, USA). 


\subsection{Cholinesterase Activity Measurements}

Reversible inhibition of AChE and BChE with oximes (50-200 $\mu \mathrm{M})$ was evaluated in the presence of the substrate concentration range ATCh $(0.1-0.7 \mathrm{mM})$ in $0.1 \mathrm{mM}$ phosphate buffer. Enzyme activity was measured by the Ellman method [35] at $25^{\circ} \mathrm{C}$ and $412 \mathrm{~nm}$ on a Tecan Infinite M200PRO plate reader (Tecan Austria, GmbH, Salzburg, Austria). Due to the low solubility, a $100 \mathrm{mM}$ stock solution of the tested compounds with the following aliquots was prepared in DMSO, so the same solvent was used in controls as well. The inhibition constants were evaluated as previously described [36].

For the reactivation of OP-inhibited cholinesterases, the enzyme was incubated with 10-fold excess sarin, cyclosarin, VX, or tabun concentrations for about $60 \mathrm{~min}$ to achieve a $95-100 \%$ inhibition rate. The inhibition mixture was fractionated on a Sephadex G-50 spin column (Roche Diagnostic GmbH, Mannheim, Germany) to remove the unconjugated nerve agent. The reactivation mixture, containing oxime $(0.1 \mathrm{mM})$ in a $0.1 \mathrm{M}$ sodium phosphate buffer $\mathrm{pH}$ 7.4, was combined with an inhibited enzyme for the initiation of the reactivation reaction. At specified time intervals, an aliquot was 100 -fold diluted in a $0.1 \mathrm{M}$ sodium phosphate buffer $\mathrm{pH} 7.4$ and upon the addition of the substrate ATCh $(1 \mathrm{mM})$ and the reagent DTNB $(0.3 \mathrm{mM})$, residual enzyme activity was measured by the Ellman method [35] at $25^{\circ} \mathrm{C}$ and $412 \mathrm{~nm}$ on a spectrophotometer (CARY 300, Varian Inc., Mulgrave,Australia). Activity was monitored up to $24 \mathrm{~h}$ with consistent correction for the inhibitory effect of oximes and the oxime-induced hydrolysis of ATCh. The firstorder reactivation rate constants $k_{\mathrm{obs}}$ at given oxime concentrations were determined by non-linear regression as described earlier [37].

\subsection{Docking of Thienostilbene Oximes}

The model system for the docking study was prepared using the geometry of the active site of butyrylcholinesterase, taken from the PDB structure 3DJY [38], where the inhibitor tabun was replaced by cyclosarin, as described above. The docking of the oximes into the active site of butyrylcholinesterase ( 15 residues) was performed using the Autodock program suite [39]. The presented results were obtained using the Lamarckian Genetic Algorithm, with a maximum of 2.5 million energy evaluations for docking calculations. The number of requested genetic algorithm dockings was 10, and 10 binding poses for each ligand were obtained, resulting in different structures of the complex between the cyclosarin-inhibited active site and the potential reactivator. Scanning of PES was carried out using the Gaussian09 program package [40], at the B3LYP/6-31G(d) level of theory.

\subsection{In Silico Prediction of ADME Properties}

In silico predictions of ADME properties were performed with $\mathrm{ACD} /$ Percepta (ver. 14.2.0; Build 2977; ACD/Laboratories, Toronto, ON, Canada).

\section{Conclusions}

This paper presents the design and synthesis of new, uncharged 2-thienostilbene oximes by an economical three-step synthesis using the Wittig reaction, Vilsmeier formylation, and the transformation of aldehydes into oximes in high yields. The expected targeted products were pure cis- and trans-isomers of syn- and anti-oximes containing different substituents bound in the para-position of the benzene ring. Finally, in each oxime formation, although four isomers were expected, cis, syn, trans,syn-, cis, anti-, and trans, anti-, considering the configuration of the carbon-carbon and carbon-nitrogen double bonds, after successive column and thin layer chromatography of the reaction mixtures, only some of isomers 13-18 were successfully isolated. Out of the eight oximes selected for the reactivation of nerve-agent inhibited cholinesterases, trans,anti-15 and trans,anti-14 and their trans,syn-counterparts reactivated cyclosarin-inhibited $\mathrm{BChE}$ up to $70 \%$. This is the first study to show the potential of thienostilbene oximes as therapeutics in OP poisoning, and it seems that further design of the compounds e.g., with amide, $\mathrm{OH}$, mono- 
and dimethylamino groups, or a triazole ring, could provide a new platform for further antidote and scavenger development for exposure to organophosphates.

Supplementary Materials: The following are available online at https://www.mdpi.com/article/ 10.3390/ph14111147/s1: Spectra contributions: ${ }^{1} \mathrm{H}$ and ${ }^{13} \mathrm{C}$ NMR spectra of the new investigated compounds, along with the 2D NMR spectra and mass spectra of all new isolated oxime derivatives 13-18; Figure S1: Superposition of the cyclosarin-bound AChE (3ZLU) and BChE (3DJY) with cyclosarin in the same conformation at the active serine; Figure S2: Superposition of cyclosarinbound AChE (3ZLU) and BChE (3DJY) with cyclosarin bound at the active serine obtained by replacing the dimethylamino and ethoxy groups of tabun with methyl and cyclohexyloxy groups of cyclosarin, respectively; Figure S3: Energy profile for the incremental decrease of the distance between the oxygen of oxime and phosphorus; Table S1: Data obtained by scanning of PES, presented in Figure S3.

Author Contributions: Conceptualization, I.Š. and Z.K.; methodology, M.M. and T.Č.; formal analysis, Ž.M. and K.L.; investigation, M.M., T.Č., D.B., I.P. and A.R.; resources, I.Š. and Z.K.; writingoriginal draft preparation, I.Š., Z.K., T.Č. and D.B.; writing-review and editing, M.M., T.Č., D.B., A.R., Z.K. and I.Š. All authors have read and agreed to the published version of the manuscript.

Funding: This research and the APC was funded by the Croatian Science Foundation (project IP2018-01-7683). The University of Zagreb's short term scientific support (2020) under the project Preparation of new annelated heteropolycyclic systems is gratefully acknowledged.

Institutional Review Board Statement: Not applicable.

Informed Consent Statement: Not applicable.

Data Availability Statement: Data is contained within the article and Supplementary Material.

Acknowledgments: The authors are grateful to Zoran Radić and Florian Nachon for a generous gift of enzymes. The authors thank Makso Herman for language editing. We also gratefully acknowledge the NMR Centre at RBI for recording all the NMR spectra.

Conflicts of Interest: The authors declare no conflict of interest.

\section{References}

1. Dolgin, E. Syrian gas attack reinforces need for better anti-sarin drugs. Nat. Med. 2013, 19, 1194. [CrossRef]

2. Steindl, D.; Boehmerle, W.; Körner, R.; Praeger, D.; Haug, M.; Nee, J.; Schreiber, A.; Scheibe, F.; Demin, K.; Jacoby, P.; et al. Novichok nerve agent poisoning. Lancet 2021, 397, 249-252. [CrossRef]

3. Darvesh, S.; Hopkins, A.D.; Geula, C. Neurobiology of Butyrylcholinesterase. Nat. Rev. Neurosci. 2003, 4, 131-138. [CrossRef] [PubMed]

4. Giacobini, E. Butyrylcholinesterase: Its Function and Inhibitors, 1st ed.; Martin, D., Ed.; Taylor \& Francis Group plc: London, UK, 2003; ISBN 1841842095.

5. Čadež, T.; Kovarik, Z. Advancements in recombinant technology for production of butyrylcholinesterase, a bioscavenger of nerve agent. Period. Biol. 2020, 121-122, 55-63. [CrossRef]

6. Sidell, F.R.; Borak, J. Chemical warfare agents: II. nerve agents. Ann. Emerg. Med. 1992, 21, 865-871. [CrossRef]

7. Timperley, C.M.; Abdollahi, M.; Al-Amri, A.S.; Baulig, A.; Benachour, D.; Borrett, V.; Cariño, F.A.; Geist, M.; Gonzalez, D.; Kane, W.; et al. Advice on assistance and protection by the Scientific Advisory Board of the Organisation for the Prohibition of Chemical Weapons: Part 2. On preventing and treating health effects from acute, prolonged, and repeated nerve agent exposure, and the identific. Toxicology 2019, 413, 13-23. [CrossRef] [PubMed]

8. Timperley, C.M.; Forman, J.E.; Abdollahi, M.; Al-Amri, A.S.; Baulig, A.; Benachour, D.; Borrett, V.; Cariño, F.A.; Geist, M.; Gonzalez, D.; et al. Advice on assistance and protection provided by the Scientific Advisory Board of the Organisation for the Prohibition of Chemical Weapons: Part 1. On medical care and treatment of injuries from nerve agents. Toxicology 2019, 415, 56-69. [CrossRef]

9. Maček Hrvat, N.; Kovarik, Z. Counteracting poisoning with chemical warfare nerve agents. Arh. Hig. Rada Toksikol. 2021, 71, 266-284. [CrossRef] [PubMed]

10. Zorbaz, T.; Kovarik, Z. Neuropharmacology: Oxime antidotes for organophosphate pesticide and nerve agent poisoning. Period. Biol. 2020, 121-122, 35-54. [CrossRef]

11. Zorbaz, T.; Mišetić, P.; Probst, N.; Žunec, S.; Zandona, A.; Mendaš, G.; Micek, V.; MačEk Hrvat, N.; Katalinić, M.; Braïki, A.; et al. Pharmacokinetic evaluation of brain penetrating morpholine-3-hydroxy-2-pyridine oxime as an antidote for nerve agent poisoning. ACS Chem. Neurosci. 2020, 11, 1072-1084. [CrossRef] 
12. Sit, R.K.; Kovarik, Z.; Maček Hrvat, N.; Žunec, S.; Green, C.; Fokin, V.V.; Sharpless, K.B.; Radic, Z.; Taylor, P. Pharmacology, pharmacokinetics, and tissue disposition of zwitterionic hydroxyiminoacetamido alkylamines as reactivating antidotes for organophosphate exposure. J. Pharmacol. Exp. Ther. 2018, 367, 363-372. [CrossRef] [PubMed]

13. Kuca, K.; Jun, D.; Musilek, K. Structural requirements of acetylcholinesterase reactivators. Mini Rev. Med. Chem. 2006, 6, 269-277. [CrossRef] [PubMed]

14. Norrahim, M.N.F.; Ahmad Shah, N.A.; Jamal, S.H.; Yunus, W.M.Z.W.; Ernest, V.F.K.V.; Kasim, N.A.M. Nanocellulose-based filters as novel barrier systems for chemical warfare agents. Solid State Phenom. 2021, 317, 180-186. [CrossRef]

15. de Koning, M.C.; van Grol, M.; Noort, D. Peripheral site ligand conjugation to a non-quaternary oxime enhances reactivation of nerve agent-inhibited human acetylcholinesterase. Toxicol. Lett. 2011, 206, 54-59. [CrossRef] [PubMed]

16. Kliachyna, M.; Santoni, G.; Nussbaum, V.; Renou, J.; Sanson, B.; Colletier, J.P.; Arboléas, M.; Loiodice, M.; Weik, M.; Jean, L.; et al. Design, synthesis and biological evaluation of novel tetrahydroacridine pyridine- Aldoxime and -Amidoxime hybrids as efficient uncharged reactivators of nerve agent-Inhibited human acetylcholinesterase. Eur. J. Med. Chem. 2014, 78, 455-467. [CrossRef] [PubMed]

17. Šagud, I.; Škorić, I.; Šindler-Kulyk, M. Excited state transformations of heterostilbenes: Pathways to polycyclic skeleta. Comptes Rendus. Chim. 2018, 21, 1043-1052. [CrossRef]

18. Ribeiro, T.S.; Prates, A.; Alves, S.R.; Oliveira-Silva, J.J.; Riehl, C.A.S.; Figueroa-Villar, J.D. The effect of neutral oximes on the reactivation of human acetylcholinesterase inhibited with paraoxon. J. Braz. Chem. Soc. 2012, 23, 1216-1225. [CrossRef]

19. Porcheddu, A.; Giacomelli, G. Synthesis of Oximes and Hydroxamic Acids. In The Chemistry of Hydroxylamines, Oximes and Hydroxamic Acids; Liebman, J.F., Rappoport, Z., Eds.; John Wiley \& Sons: New York, NY, USA, 2009; pp. $163-231$.

20. Alavijeh, M.S.; Chishty, M.; Qaiser, M.Z.; Palmer, A.M. Drug metabolism and pharmacokinetics, the blood-brain barrier, and central nervous system drug discovery. NeuroRX 2005, 2, 554-571. [CrossRef]

21. Testa, B.; Crivori, P.; Reist, M.; Carrupt, P.A. The influence of lipophylicity on the pharmacokinetic behavior of drugs: Concepts and examples. Perspect. Drug Discov. Des. 2000, 19, 179-211. [CrossRef]

22. Pajouhesh, H.; Lenz, G.R. Medicinal chemical properties of successful central nervous system drugs. NeuroRX 2005, 2, 541-553. [CrossRef] [PubMed]

23. Stocks, M. The small molecule drug discovery process-from target selection to candidate selection. In Introduction to Biological and Small Molecule Drug Research and Development: Theory and Case Studies; Jefferis, R., Roberts, S., Ganellin, R., Eds.; The University of Nottingham: England, UK, 2013; pp. 81-126. [CrossRef]

24. Zandona, A.; Katalinić, M.; Šinko, G.; Radman Kastelic, A.; Primožič, I.; Kovarik, Z. Targeting organophosphorus compounds poisoning by novel quinuclidine-3 oximes: Development of butyrylcholinesterase-based bioscavengers. Arch. Toxicol. 2020, 94, 3157-3171. [CrossRef]

25. Artursson, E.; Andersson, P.O.; Akfur, C.; Linusson, A.; Borjegren, S.; Ekstrom, F. Catalytic-site conformational equilibrium in nerve-agent adducts of acetylcholinesterase; possible implications for the HI-6 antidote substrate specificity. Biochem. Pharmacol. 2013, 85, 1389-1397. [CrossRef] [PubMed]

26. Cuya, T.; da Silva Gonçalves, A.; da Silva, J.A.V.; Ramalho, T.C.; Kuca, C.C.K.; França, T.C.C. The role of the oximes HI-6 and HS-6 inside human acetylcholinesterase inhibited with nerve agents: A computational study. J. Biomol. Struct. Dyn. 2018, 36, 3444-3452. [CrossRef] [PubMed]

27. Maraković, N.; Knežević, A.; Rončević, I.; Brazzolotto, X.; Kovarik, Z.; Šinko, G. Enantioseparation, in vitro testing, and structural characterization of triple-binding reactivators of organophosphate-inhibited cholinesterases. Biochem. J. 2020, 477, 2771-2790. [CrossRef] [PubMed]

28. Kumar, S.; Pandey, A.K.; Singh, R.; Singh, K.N. Decarboxylative arylation of $\alpha, \beta$-unsaturated carboxylic acids using aryl trazenes by copper/ionic liquid combination in PEG-400. Eur. J. Org. Chem. 2018, 2018, 5942-5949. [CrossRef]

29. Qu, J.; Cao, C.T.; Cao, C. Determining the excited-state substituent constants of furyl and thienyl groups. J. Phys. Org. Chem. 2018, 31, 3799. [CrossRef]

30. Lee, S.K.; Min, H.Y.; Huh, K.S.; Kim, E.Y.; Lee, E.; Song, S.; Kim, S. Styrylheterocycles: A novel class of inhibitors on lipopolysaccharide-induced nitric oxide production. Bioorganic Med. Chem. Lett. 2003, 13, 3689-3692. [CrossRef]

31. Jin, J.; Li, X.; Zhang, J.; Zhao, P.; Tian, H. Rational design of double-check mercury ion chemosensors based on photochromic compounds. Isr. J. Chem. 2013, 53, 288-293. [CrossRef]

32. Jones, G.; Stanforth, S.P. The Vilsmeier reaction of fully conjugated carbocycles and heterocycles. Org. React. 1997, 49, 1-330.

33. Yoshimura, S.; Takahashi, S.; Kawamata, A.; Kikugawa, K.; Suehiro, H.; Aoki, A. Synthesis of $\alpha$-substituted alkanoic acids and inhibition of platelet aggregation. Chem. Pharm. Bull. 1978, 26, 685-702. [CrossRef]

34. Jiang, X.; Yang, X.; Zhao, C.; Jin, K.; Sun, L. Electrogenerated chemiluminiscence of a series of donor-acceptor molecules and X-ray crystallographic evidence for the reaction mechanisms. J. Phys. Chem. C 2007, 111, 9595-9602. [CrossRef]

35. Ellman, G.L.; Courtney, K.D.; Andres, V.; Featherstone, J.; Featherstone, R.M. A new and rapid colorimetric determination of acetylcholinesterase activity. Biochem. Pharmacol. 1961, 7, 88-95. [CrossRef]

36. Kovarik, Z.; Ciban, N.; Radić, Z.; Simeon-Rudolf, V.; Taylor, P. Active site mutant acetylcholinesterase interactions with 2-PAM, HI-6, and DDVP. Biochem. Biophys. Res. Commun. 2006, 342, 973-978. [CrossRef] [PubMed]

37. Maček Hrvat, N.; Zorbaz, T.; Šinko, G.; Kovarik, Z. The estimation of oxime efficiency is affected by the experimental design of phosphylated acetylcholinesterase reactivation. Toxicol. Lett. 2018, 293, 222-228. [CrossRef] [PubMed] 
38. Carletti, E.; Li, H.; Li, B.; Ekstrom, F.; Nicolet, Y.; Loiodice, M.; Gillon, E.; Froment, M.T.; Lockridge, O.; Schopfer, L.M.; et al. Aging of cholinesterases phosphylated by tabun proceeds through O-dealkylation. J. Am. Chem. Soc. 2008, 130, 16011-16020. [CrossRef]

39. Morris, G.M.; Huey, R.; Lindstrom, W.; Sanner, M.F.; Belew, R.K.; Goodsell, D.S.; Olson, A.J. AutoDock4 and AutoDockTools4: Automated docking with selective receptor flexibility. J. Comput. Chem. 2009, 16, 2785-2791. [CrossRef]

40. Frisch, M.J.; Trucks, G.W.; Schlegel, H.B.; Scuseria, G.E.; Robb, M.A.; Cheeseman, J.R.; Scalmani, G.; Barone, V.; Petersson, G.A.; Nakatsuji, H.; et al. Gaussian 09; Revision D.01; Gaussian, Inc.: Wallingford, CT, USA, 2009. 